INTER NATIONAL MONETARY FUND
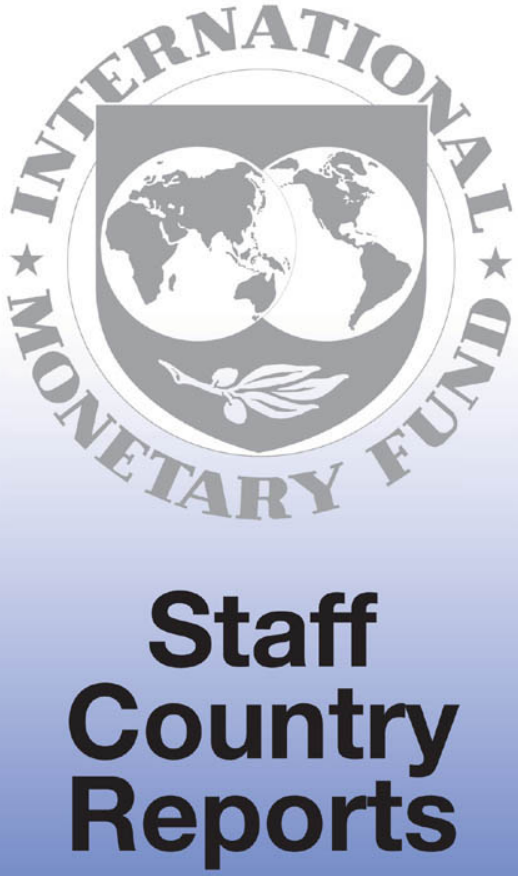


\section{Rwanda - Second Review Under the Three-Year Arrangement Under the Poverty Reduction and Growth Facility, Request for Waiver of Nonobservance of Performance Criterion, and Modification of Performance Criteria-Staff Report; Press Release on the Executive Board Discussion; and Statement by the Executive Director for Rwanda}

In the context of the second review under the three-year arrangement under the Poverty and Growth Facility, request for waiver of nonobservance of a performance criterion, and modification of performance criteria, the following documents have been released and are included in this package:

- $\quad$ the staff report for the Second Review Under the Three-Year Arrangement Under the Poverty Reduction and Growth Facility, Request for Waiver of Nonobservance of Performance Criterion, and Modification of Performance Criteria, prepared by a staff team of the IMF, following discussions that ended on April 3, 2007, with the officials of Rwanda on economic developments and policies. Based on information available at the time of these discussions, the staff report was completed on June 7, 2007. The views expressed in the staff report are those of the staff team and do not necessarily reflect the views of the Executive Board of the IMF;

- $\quad$ a Press Release summarizing the views of the Executive Board as expressed during its June 25, 2007 discussion of the staff report that complete the review; and

a statement by the Executive Director for Rwanda.

The documents listed below have been or will be separately released.

Letter of Intent sent to the IMF by the authorities of Rwanda*

Memorandum of Economic and Financial Policies by the authorities of Rwanda*

Technical Memorandum of Understanding*

*Also included in the Staff Report

The policy of publication of staff reports and other documents allows for the deletion of market-sensitive information.

To assist the IMF in evaluating the publication policy, reader comments are invited and may be sent by e-mail to publicationpolicy@imf.org.

Copies of this report are available to the public from

International Monetary Fund $\bullet$ Publication Services

$70019^{\text {th }}$ Street, N.W. • Washington, D.C. 20431

Telephone: (202) 623-7430 • Telefax: (202) 623-7201

E-mail: publications@imf.org •Internet: http://www.imf.org

Price: $\$ 18.00$ a copy

International Monetary Fund

Washington, D.C.

CInternational Monetary Fund. Not for Redistribution 
This page intentionally left blank 
INTERNATIONAL MONETARY FUND

RWANDA

\title{
Second Review Under the Three-Year Arrangement Under the Poverty Reduction and Growth Facility, Request for Waiver of Nonobservance of Performance Criterion, and Modification of Performance Criteria
}

\author{
Prepared by the African Department
}

(In collaboration with other departments)

Approved by Sharmini Coorey and Mark Plant

June 7, 2007

- $\quad$ PRGF/debt relief. The Executive Board approved a PRGF arrangement with access of 10 percent of quota on June 5, 2006, and completed the first review on January 29, 2007. Rwanda reached the completion point for the enhanced HIPC Initiative in April 2005 and received MDRI debt relief in January 2006.

- Main topics. Performance in 2006 was broadly satisfactory. The challenge for 2007 is to successfully manage a grant-financed fiscal expansion and pave the way for possible scaling up of aid in the future. The report recommends completion of the second review of the PRGF arrangement.

- $\quad$ Participants. The mission met with Minister of Finance and Economic Planning Musoni, Governor Kanimba of the National Bank of Rwanda, other government officials, and representatives of the private sector and the international community. The mission team headed by Ms. Kostial consisted of Mr. Darius, Mr. Mitchell, and Ms. Kaendera (all AFR) and was assisted by Mr. Engström, the resident representative. 


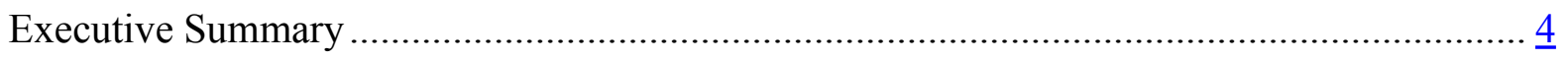

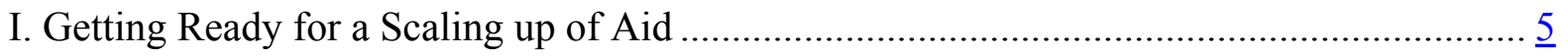

II. The Program Is on Track but Warrants Close Monitoring................................................. $\underline{5}$

III. Macroeconomic Challenge: Large Fiscal Expansion is Uncharted Territory ..................... $\mathbb{Z}$

A. Fiscal Policy—Focused on Priorities and a Smooth Spending Path................................. $\underline{9}$

B. Monetary Policy —Adapting to Changes in Money Demand ………………………... 10

C. Exchange Rate Policy — Gradual Changes .................................................................. 10

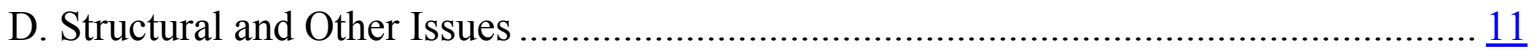

IV. Prospects For Scaling up are Uncertain.................................................................... 13

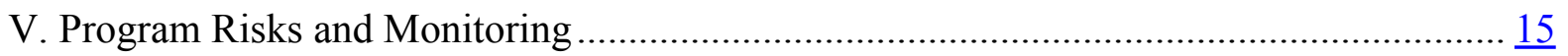

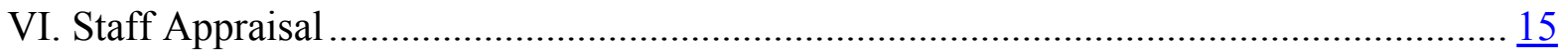

Box

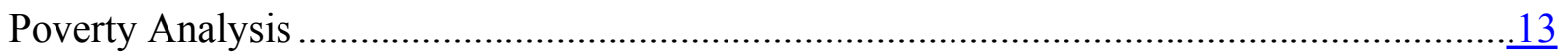

Tables

1. Selected Economic and Financial Indicators, 2003-07 ……...................................17

2. Operations of the Central Government, 2005-07 ……........................................

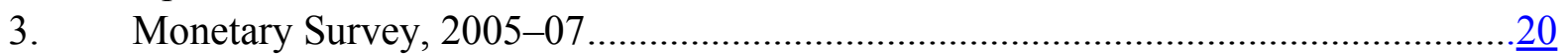

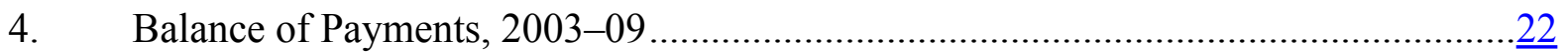

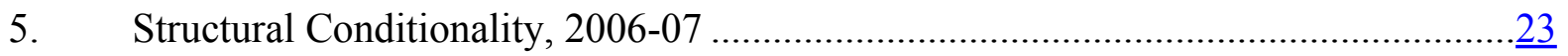

6. Proposed Schedule of Disbursement under the PRGF Arrangement, 2007-09 ….......24

7. Modification of end-June 2007 Performance Criteria on the Domestic Fiscal Balance and Net Credit to Government ................................................................... 
Appendices

I. Letter of Intent

Attachment I. Memorandum of Economic and Financial Policies of of the Government of Rwanda(2007)

Attachment II. Technical Memorandum of Understanding ...................................41

II. Millennium Development Goals.........................................................................4 


\section{EXECUTIVE SUMMARY}

Program performance was broadly satisfactory. Growth at over 5 percent in 2006 was higher than expected, though inflation at 12 percent also exceeded the program target. Inflation declined slightly through April 2007, but inflation excluding food and energy items picked up to over 6 percent since February after holding steady at 5 percent for several years. Although fiscal policy stayed on track, delays in the reimbursements from the African Union (AU) for Rwanda's peacekeeping efforts meant that the December 2006 performance criterion on net credit to government was missed. Though monetary targets were met, the "fit" of the monetary program deteriorated because of an increase in money demand. Structural performance was less satisfactory: all three benchmarks were missed. Two (issuing accounting instructions and publication of a fertilizer strategy) have since been implemented and the third (review of the wage structure) has been converted into a new benchmark for September 2007.

Rwanda must prepare for a possible scaling up of aid. This entails managing a grantfinanced fiscal expansion of 3 percent in 2007 without jeopardizing macroeconomic stability. To prevent a crowding out of the private sector, indicative limits on domestic debt have been established. If these limits are exceeded or inflation is rekindled, the domestic component of fiscal spending must be released more gradually. Preparing for scaling up will also require improving outcome indicators because success in reducing poverty has so far been limited. To this end, the new PRSP is expected to improve monitoring and to emphasize raising agricultural yields to address rural poverty. To ensure that development spending is not crowded out by peace keeping efforts, the authorities are seeking timely payments from the AU.

To account for higher money demand, the monetary program for 2007 has been rebased. Reserve money growth will be limited through sales of foreign exchange.

The main obstacle to exchange rate flexibility is a lack of expertise in this area among banks and their clients. This will be addressed over time by a gradual move toward an interbank market for foreign exchange and by attracting international banks as the banking sector consolidates.

Though prospects for scaling up are encouraging, the timing and order of magnitude are uncertain. The authorities stressed their commitment to keep debt sustainable but noted that, if scaling up does not materialize soon, they would consider concessional borrowing for projects with a high rate of return.

On the structural side, the focus remains on public financial management (PFM) and the financial sector. PFM reforms, particularly for local governments, must be accelerated. The authorities' financial sector development plan is a sound basis for building long-term financial markets.

The primary program risk is that the fiscal expansion will not be properly managed and inflation would be rekindled. 


\section{GETTING READY FOR A SCALING UP OF AID}

1. Rwanda must prepare for a possible scaling up of aid. In 2007 it expects to receive additional grants from the Fast Track Education Initiative (FTEI) and the African Development Bank (AfDB). Prospects for scaling up in the medium term are good, though amounts and timing are uncertain. To pave the way for scaling up, Rwanda must

- $\quad$ preserve macro stability while accommodating a grant-financed fiscal expansion; and

- $\quad$ improve outcome indicators by making the most productive use of resources.

\section{The Program Is on Track but Warrants Close Monitoring}

\section{Though growth in 2006 was} good, inflationary pressures have emerged. Despite depressed agricultural production, GDP growth was buoyed up, to over 5 percent, by the manufacturing and services sectors. Inflation picked up mid-year and since December 2006 has hovered around 12 percent. Though most of the increase comes from food and energy items, inflation excluding these increased to over 6 percent since

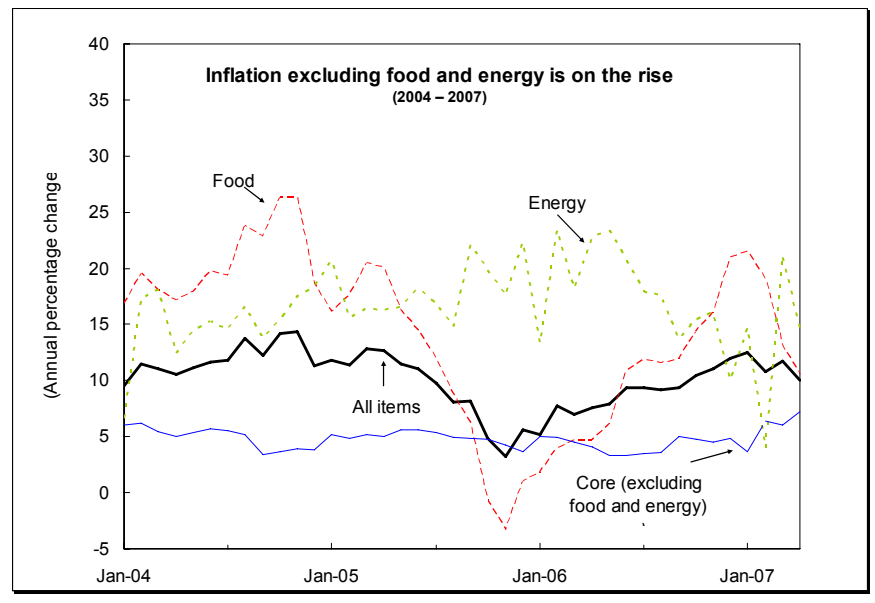
February after holding steady at about 5 percent since 2004 .

3. Fiscal policy was broadly in line with the program through March 2007. In 2006 revenue overperformance financed unforeseen outlays, mostly for priorities. As foreshadowed in Country report No. 07/80, the end-2006 target on net credit to government was not met because reimbursements from the African Union (AU) for Rwanda's peacekeeping in Darfur were delayed. Preliminary data for the first quarter of 2007 indicate that the targets on net credit to the government and the domestic fiscal balance were exceeded because grants from the World Bank and the European Union (EU) were delayed; these will come in the second quarter. ${ }^{1}$

\section{With an increase in money demand, the fit of the monetary program} deteriorated. Broad money exceeded indicative limits during 2006 by a wide margin,

\footnotetext{
${ }^{1}$ Parliamentary approval of the Poverty Reduction Support Grant took more time than envisaged and the EU decided to merge two tranches. The program has an automatic adjuster requiring a reduction in spending as a safeguard for permanent grant shortfalls; given that the delay was temporary, the authorities, in consultation with staff, decided against curtailing spending.
} 
because of an increase in private sector credit intended mainly for investment and higher net foreign assets of commercial banks reflecting inflows. To meet the end-2006 reserve money target, the National Bank of Rwanda (NBR) reduced commercial bank reserves substantially below the legal reserve requirement for one day. ${ }^{2}$ Following consultations with staff, the NBR allowed the end-March 2007 reserve money target to be breached, given the continued strong money demand. ${ }^{3}$
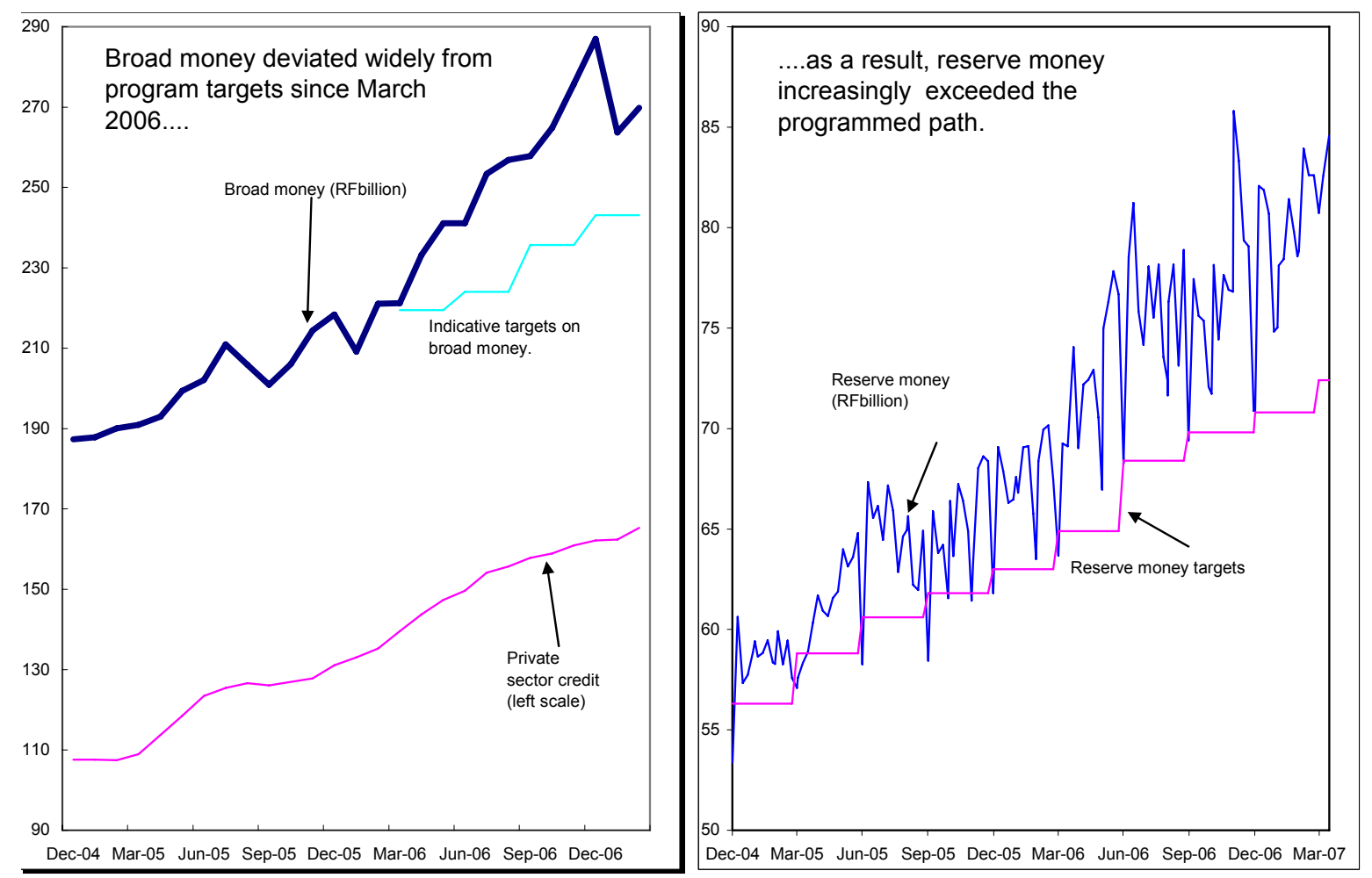

Source: Rwandese authorities and IMF staff.

\footnotetext{
${ }^{2}$ The requirement is measured as an average over 2 weeks.

${ }^{3}$ The monetary program for 2007 has now been rebased (\$14).
} 
5. The real exchange rate again appreciated moderately in 2006. The NBR received higher than expected inflows for projects, which were mostly used for domestic purchases. To prevent a sharp appreciation, it resorted to heavy sterilization in the last quarter by issuing domestic debt. International reserves substantially exceeded program targets, reaching $5 \frac{1}{2}$ months of imports at end2006 .

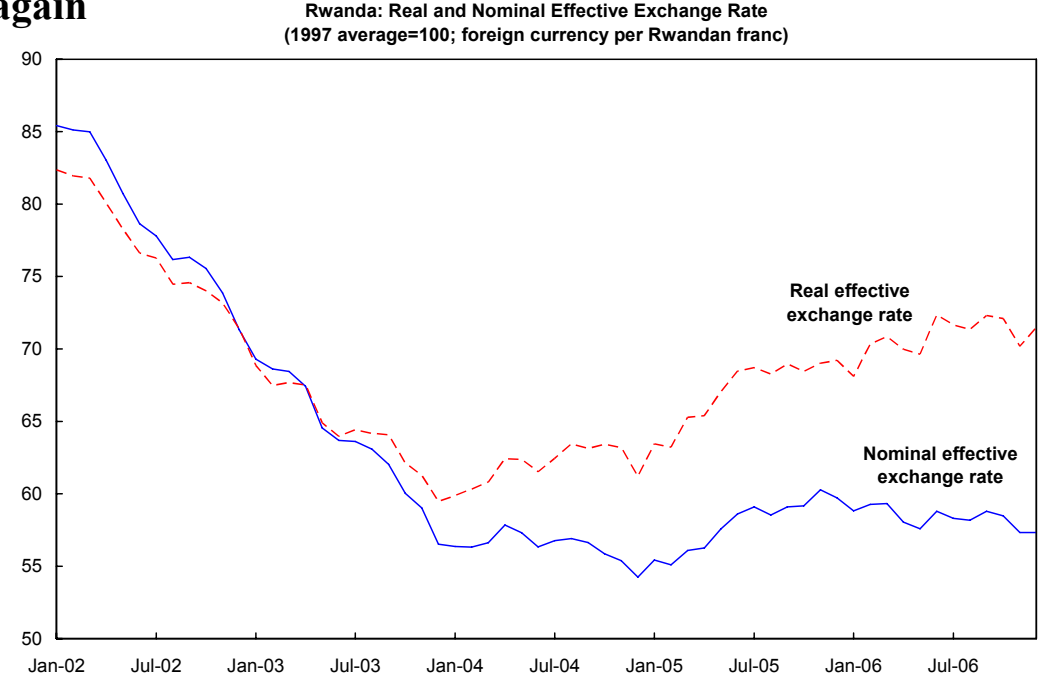

6. Adherence to quantitative conditionality for the second review was good but structural performance was less satisfactory. Except for the limit on net credit to government all quantitative end-2006 performance criteria (PCs) were met (Table 1 of the Memorandum of Economic and Financial Policies (MEFP) $)^{4}$ ). All three structural benchmarks were missed. The one on issuing accounting instructions (December 2006) was implemented in May and the one on publishing a fertilizer distribution strategy (February 2007) in April. The third, a review of the wage structure of the civil service (December 2006), has been converted into a benchmark for September 2007 because the authorities felt that technical assistance was needed.

\section{Macroeconomic Challenge: Large Fiscal Expansion is UnCharted TERRITORY}

7. The program allows for a large fiscal expansion. It originally envisaged an increase from 2006 in the domestic deficit (excluding spending on demobilization and peacekeeping) by 1.3 percent of GDP. Staff proposes a further widening of the deficit by up to 2 percent of GDP reflecting:

- $\quad 1.2$ percent of GDP in additional education spending funded by grants mainly from the FTEI.

- $\quad 0.5$ percent of GDP for contingent spending. So far, additional grants of 0.1 percent of GDP have been identified and related spending incorporated in the program.

\footnotetext{
${ }^{4}$ The authorities describe their policies in the MEFP.
} 
The program would automatically accommodate further contingent spending up to 0.4 percent of GDP should more grants materialize. ${ }^{5}$

- $\quad 0.3$ percent of GDP in food imports to bridge the period until the next harvest (given shortages of storable crops), financed by a drawdown in international reserves. ${ }^{6}$

8. With such expansion being uncharted territory, close monitoring is needed. The authorities introduced a safeguard - indicative limits on consolidated domestic debt of the central government and the NBR - to avoid crowding out the private sector and thus ensure a real resource transfer through an increase in net imports ("spend and absorb," $\mid 15)$. They will release the domestic component of spending more gradually if the limits are exceeded or inflation is rekindled (MEFP, 111 ). Staff noted that a moderate pickup in inflation beyond the program target was possible to absorb increased aid from the fiscal expansion, but emphasized that an acceleration must be prevented.

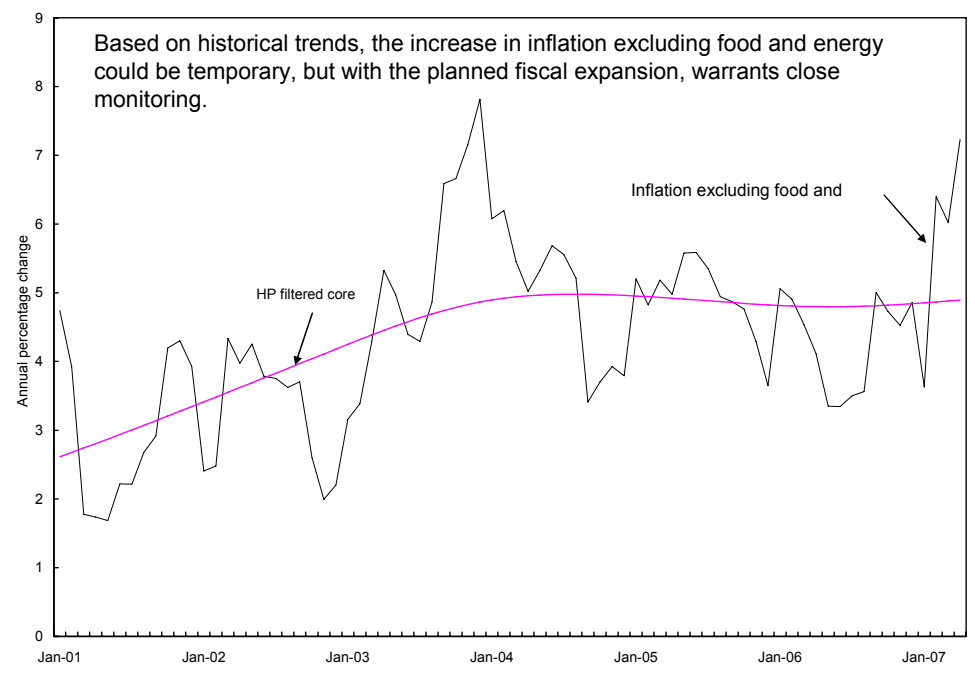

9. The macroeconomic framework for $\mathbf{2 0 0 7}$ is broadly unchanged. It envisages a growth rate of $4 \frac{1}{2}-6 \frac{1}{2}$ percent, inflation of 5 percent, and international reserves of about 5 months of imports. On the upside, there could be a supply response if all domestic spending is released. On the downside, with the first 2007 harvest below the 2006 level, agricultural production could be depressed and put upward pressure on inflation.

\footnotetext{
${ }^{5}$ Should grants turn out even higher, an increase in the deficit beyond the revised target would be discussed during the next review.

${ }^{6}$ The program automatically accommodates food imports.
} 


\section{A. Fiscal Policy_Focused on Priorities and a Smooth Spending Path}

\section{The authorities will keep the revenue ratio, excluding one-off items, at the 2006}

level. Revenue in the last quarter of 2006 was boosted by one-off sales of government assets and arrears collection. If there are revenue shortfalls, the authorities would raise petroleum excises (MEFP, $\llbracket 13$ ).

\section{New spending would be almost entirely for priorities, primarily related to} education (MEFP, $\uparrow 14$ for details). Increases in nonpriority spending reflect an underestimate of the domestic interest bill and outlays for resettling refugees from Tanzania; they are financed by higher than programmed revenue.

\section{Staff proposes to relax the end-June limits on the domestic fiscal balance and net} credit to government to take account of a timing issue. ${ }^{7}$ A grant from the AfDB has been delayed because the new PRSP, one of the triggers for the grant, needs to incorporate new information ( $\mid 23)$. The relaxation would prevent a disruption in spending because the program includes an automatic reduction in the limits on the deficit and domestic financing to grant shortfalls (footnote 1). The relaxation also accommodates unforeseen outlays in the first half of the year, including the government's temporary acquisition of 50 percent of the shares in the largest commercial bank, Banque de $\operatorname{Kigali}^{8}$ (BK) (MEFP, $\uparrow 12$ plus table and 『19). End-September and end-year targets are not affected because the delayed grants are expected to come in the third quarter while higher revenue, additional grants, and proceeds from the sale of $\mathrm{BK}^{9}$ will finance the new outlays (MEFP, $\left.\mid 24\right)$. Staff considers the relaxation justifiable because it is temporary and the spending path would have been too backloaded otherwise. Should delays in AU reimbursements for Darfur persist, the authorities will curtail other spending to meet the limits on net credit to government (MEFP, $\mid 15$ ). The AU has been consistently late in its payments to Rwanda and efforts by the authorities to receive reimbursement for amounts owed have met with limited success. This crowds out spending for Rwanda's development. Staff urged the authorities to persevere in their efforts to receive timely future payments. ${ }^{10}$

13. Spending pressures from decentralization must be monitored. The 2007 program envisages almost a doubling of funds channeled through local governments. Most local

\footnotetext{
${ }^{7}$ By 1.3 percent of GDP and 0.9 percent of GDP, respectively (Table 7).

${ }^{8}$ The Belgian bank holding the shares decided to sell them after it was acquired by Fortis, a European financial service provider.

${ }^{9}$ Based on preliminary indications of interest in BK, a Fund technical assistance advisor considered this time table as possible.

${ }^{10}$ The UN may take over the peace keeping activities in Darfur.
} 
governments have requested more funds, but some accumulated funds in the first quarter. To enhance transparency, structural conditionality related to publication of local government spending was added to the program (MEFP, $₫ 17$ ).

\section{B. Monetary Policy_Adapting to Changes in Money Demand}

\section{The reserve money program would be rebased to reflect the past increase in} money demand. This rebasing ensures that banks can meet the legal reserve requirement at the end of the quarter with some margin for excess reserves, given the volatility of cash balances in banks' local branches ( $\mid 4)$. To make the program more binding, the quarterly target will be measured on a monthly frequency rather than once at the end of the quarter (MEFP, \19). The authorities and staff considered more frequent monitoring but deemed it inappropriate, given considerable uncertainties in estimating money demand, which will be closely monitored.

\section{Reserve money growth will be limited by sales of foreign exchange. Domestic} debt is expected to remain at roughly the nominal 2006 level because there will be less of a need for domestic sterilization given the budget's higher import component and higher foreign exchange sales. ${ }^{11}$ Staff emphasized the need to improve liquidity forecasts by regularly reviewing the foreign exchange component of the budget and projects and encouraged the improved monitoring of project accounts (MEFP, $\llbracket 16$ ). Given that the maturity of domestic debt has shortened in the last 2 years, it welcomed plans to reissue

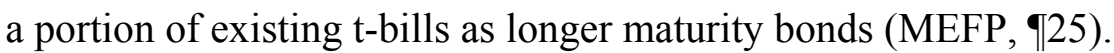

\section{Exchange Rate Policy-Gradual Changes}

\section{The NBR's foreign exchange interventions in $\mathbf{2 0 0 7}$ hinge on the budget's import}

component. Should the import component be lower than anticipated, the NBR should seek to increase foreign exchange sales while avoiding a large exchange rate appreciation, given market rigidities ( $(17)$. Staff noted that this strategy could cause a temporary breach of the domestic debt benchmark to meet reserve money limits. Any large deviation from projections of the import component would necessitate a review of the spending program.

\section{Removing obstacles to exchange rate flexibility will take time. The exchange rate} has behaved like a conventional fixed peg because the NBR and banks are concerned about the impact of a strong appreciation on exporters. The main problem is the absence of hedging instruments for exporters, such as foreign exchange lending. Banks are hesitant to take foreign exchange risk on their books because of difficulties in pricing interest rates on foreign currency loans and liquidity risks, such as early withdrawal by depositors of foreign

\footnotetext{
${ }^{11}$ If the monetary program were not rebased, domestic debt would have increased by about 0.4 percent of GDP. The stock of domestic debt has been revised upward by about 8 percent of GDP to account for reconciled and consolidated pre-genocide debt, which is noninterest bearing.
} 
currency deposits and absence of credit lines in foreign currency with foreign correspondents. Banks also noted that exporters lack capacity to manage foreign exchange liabilities. To build expertise, the NBR will

- in coordination with banks, gradually transition from the auction-based system to an interbank foreign exchange market, with technical assistance from MCM (MEFP, \20).

- $\quad$ open the banking sector to international banks (see $\llbracket 19$ ).

The authorities expect that these measures will eventually enhance exchange rate flexibility. The development of hedging instruments and capital markets together with sectoral export promotion and infrastructure investments are expected mitigate the impact on exports of a possible real appreciation from scaling up.

\section{Structural and Other Issues}

18. The focus of structural conditionality is unchanged. It focuses on PFM, poverty reduction, financial sector reform, and private sector development.

19. The NBR is appropriately reinforcing the capital base of commercial banks. The minimum capital requirement for banks will be increased on January 1, 2008. Noncompliant banks have provided plans on how they will implement the increase, including merger scenarios. Privatization of the BK is well under way, as is the recapitalization by new shareholders of a problem bank; several international banks have indicated interest in both (MEFP, $\llbracket 24)$.

20. Financial sector reforms are being extended beyond the banking sector. A Financial Sector Development Plan that was drafted in close cooperation with the World Bank and the Fund has been presented to donors for financing (MEFP, $\mathbb{q} 25$ ).

21. PFM reforms are moving more slowly than expected. Staff urged the authorities to accelerate the implementation of the Organic Budget Law (MEFP, $\uparrow 18$ ).

22. On other issues:

- $\quad$ The NBR is making good progress in strengthening its safeguards (MEFP, $\mathbb{2} 21$ ).

- The recent rebasing of the national accounts is welcome, but the GDP deflator needs to be refined (MEFP, $\uparrow 28$ ). 

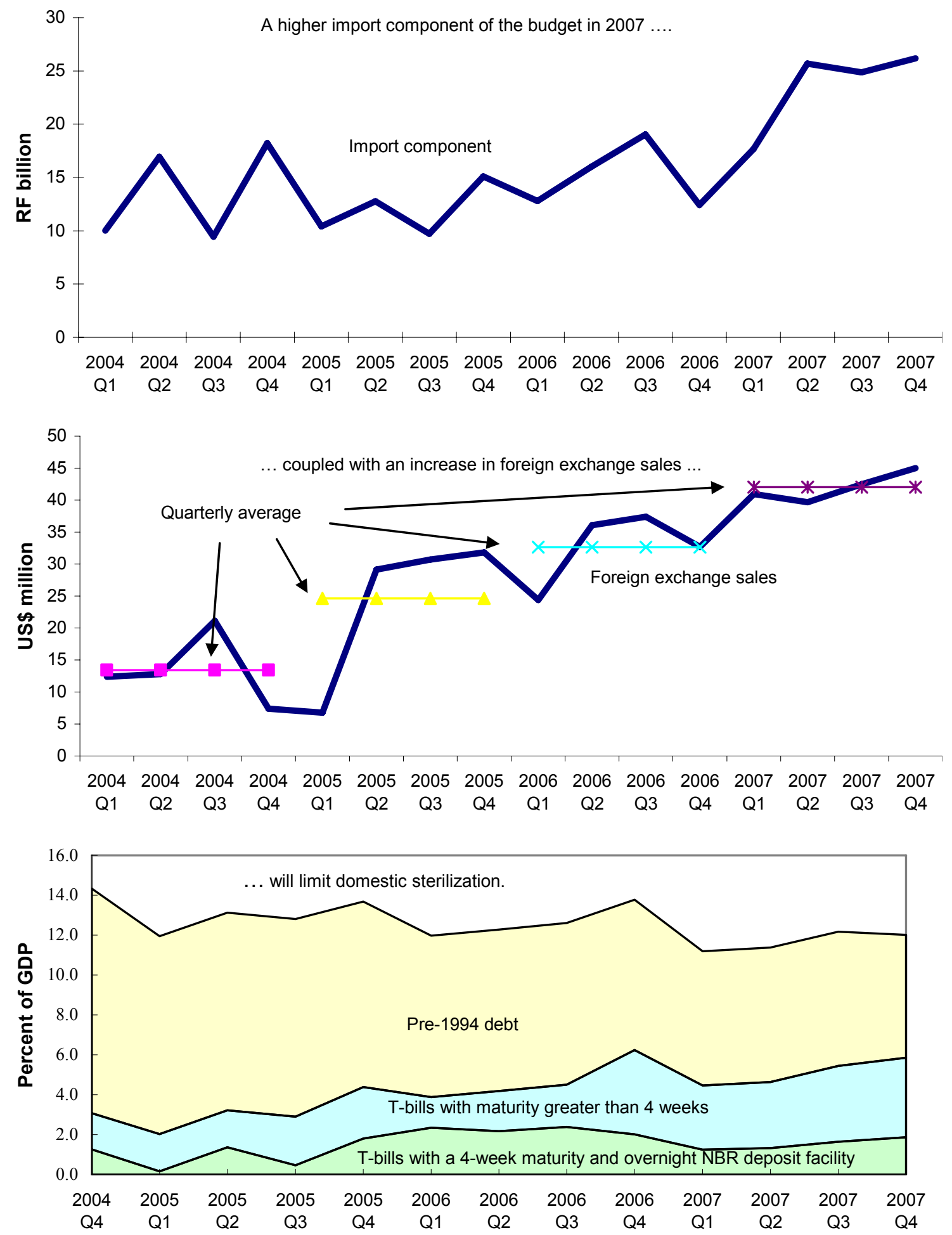

Source: Rwandese authorities and IMF staff estimates. 


\section{Prospects For Scaling UP ARE UnCERTAIN}

23. Poverty reduction must accelerate. Because success in reducing poverty has been limited (Box 1), the authorities are reviewing policies and outcome indicators. Because this will take time, the PRSP update is expected to be finalized only before year-end. An integral component of the revised strategy will be to raise agricultural yields and address population growth (MEFP, $\uparrow 8$ ).

\section{Box 1. Poverty Analysis}

Recent socioeconomic surveys found that

- High growth rates have not substantially reduced poverty. Poverty has a low income elasticity of 0.4 ; the average for developing countries is 1.0-2.0. As a result, poverty declined only slightly, from 60.5 percent in 2000/01 to 57 percent in 2005/06.

- Poverty varies noticeably by location. Poverty is high in densely populated areas. In addition to lack of income, poverty is caused by inadequate physical assets, such as land and livestock; poor soil; adverse weather; and inadequate infrastructure.

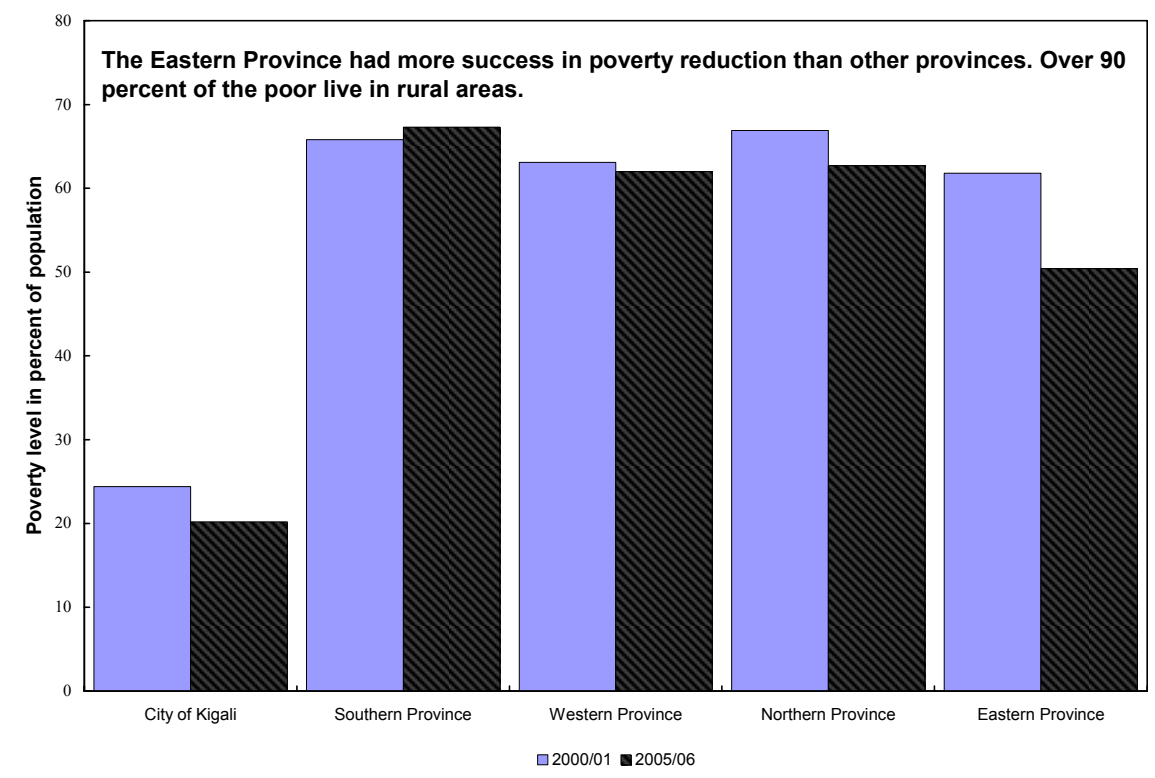

- With rising fertility and reduced mortality, population has grown at an annual average of about 3 percent.

- Social indicators have improved somewhat:

○ Primary school enrolment has increased to 86 percent, but secondary school enrolment is still only 10 percent.

○ The frequency of medical consultations has increased marginally; it is expected to increase more now that almost half the population is covered by health insurance.

O Only about two-thirds of households have access to safe drinking water. 
24. Scaled-up aid over the medium term is uncertain. FTEI funds are scheduled to increase and prospects include access to the U.S. Millennium Challenge Account (MCC), the Catalytic Fund, and AfDB and EU financing of regional infrastructure investments. The authorities stressed their commitment to keeping external debt sustainable, but if aid is not scaled up in 2008, they would consider concessional borrowing for projects that have a high rate of return such as in the energy sector (MEFP, $\mid 26$ ). They asked whether an improvement in Rwanda's Country Performance and Institutional Assessment rating could trigger a reassessment of Rwanda's risk of debt distress within the low-income-country debt sustainability framework, which in turn could raise the International Development Association allocation. Staff cautioned against a new build-up of debt and recommended that the authorities address policy weaknesses that prevented access to the MCC. ${ }^{12}$

\section{Given uncertainties, the $\mathbf{2 0 0 8}$ budget must focus on sustainability. Staff}

emphasized that it should be embedded in a medium-term framework to assess sustainability of any increases in recurrent spending. It also recommended to enhance donor coordination to make better use of funds. ${ }^{13}$

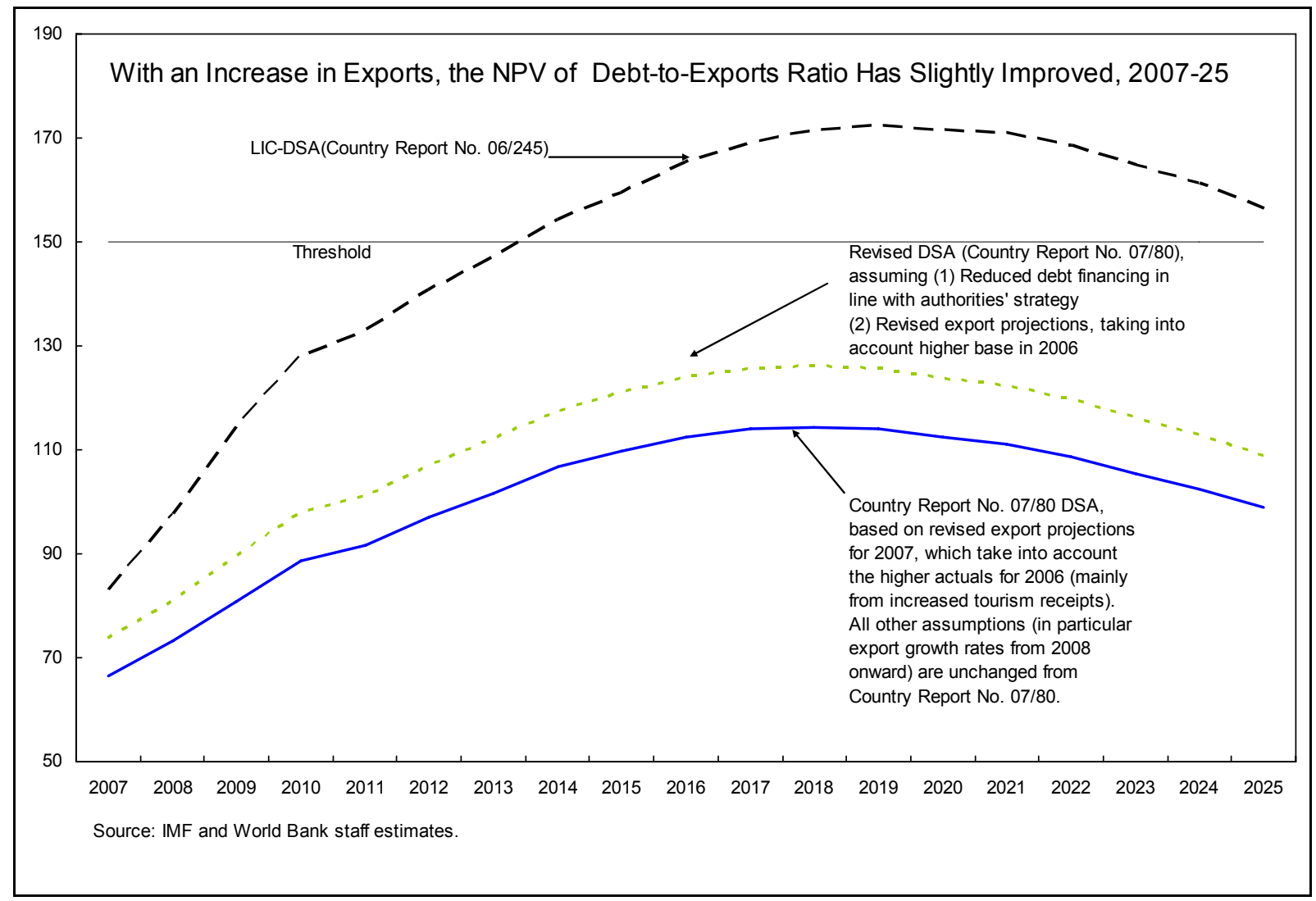

\footnotetext{
${ }^{12}$ A debt sustainability analysis will be undertaken for the preparation of the 2008 budget.

${ }^{13}$ Recent reviews of agriculture, health, and social spending found that, with a significant share of aid off budget, there is a large overhead as well as duplication and inefficient allocations between projects.
} 


\section{Program Risks AND Monitoring}

26. The program is being adapted to accommodate the grant-financed fiscal expansion but this also increases risks. The main risk is that the expansion will rekindle inflation. The authorities must also curb new spending pressures.

27. Program monitoring for 2007 is described in the MEFP ( $(9-29$ and 30) and the technical memorandum of understanding. The program design is generally the same, but an indicative target on domestic debt was added, the reserve money definition was changed, and modifications are proposed to the end-June and end-December PCs on reserve money and the end-June PCs for net credit to the government and the domestic fiscal balance.

\section{Staff Appraisal}

28. This year could be a milestone in Rwanda's development. Staff believes that a large fiscal expansion can be managed without jeopardizing macroeconomic stability. However, given uncertainties, the authorities must stand ready to make adjustments should there be an acceleration in inflation or crowding out of the private sector. The indicative limits on domestic debt provide a safeguard to protect program objectives.

29. Fiscal policies will be the linchpin for keeping the program on track. Should the budget's import component be lower than anticipated, the NBR must seek to step up foreign exchange sales. If this cannot be done without causing a large exchange rate appreciation, domestic spending must be released more gradually. Staff urges the authorities to improve liquidity forecasting and also resist any new spending pressures. Given the potential of peacekeeping efforts to crowd out other spending, the authorities should seek timely reimbursement from the AU.

30. The rebasing of the monetary program would allow the program to better reflect money demand. Nonetheless, uncertainty about money demand will continue to challenge monetary policy. The NBR must also be vigilant in monitoring inflationary developments.

31. Steps to increase exchange rate flexibility are welcome. To build expertise, staff urges the authorities to move swiftly toward an interbank foreign exchange market in coordination with banks and open the market to international banks.

32. PFM reforms must be stepped up. This is critical to ensure that funds are used for their intended purpose. With decentralization, the monitoring of local governments must be strengthened.

33. Progress in financial sector reform is encouraging. The increase in the capital requirement of banks is welcome as are prospects for banks' consolidation and the entry of foreign banks. Staff regards the FSDP as a sound basis for building long-term financial markets. 
34. The PRSP update must revamp Rwanda's poverty reduction strategy. Emphasis on increasing agricultural yields is welcome, as is better monitoring of outcome indicators.

35. Staff cautions against shifting external financing toward loans. Given Rwanda's small export base and vulnerability to shocks, the authorities should seek new sources of grants.

36. Staff recommends that the requested waiver be granted-the nonobservance was temporary - the PCs on reserve money, the domestic fiscal balance and net credit to government be modified, and that the second review of the PRGF arrangement be completed. 
Table 1: Rwanda: Selected Economic and Financial Indicators, 2003-07

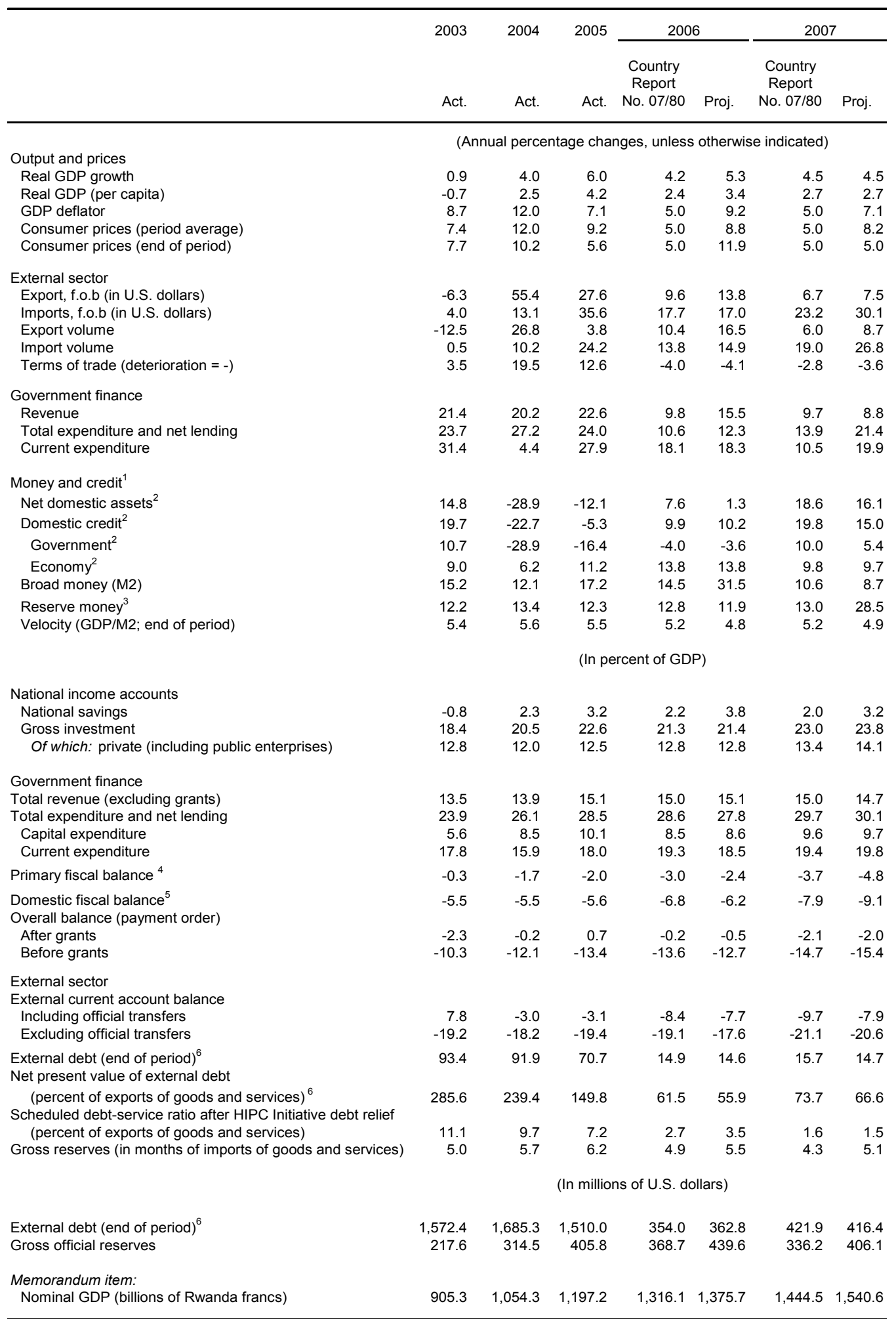

Sources: Rwandese authorities; and IMF staff estimates and projections.

${ }^{1}$ Data up to 2005 based on current exchange rates; for 2006, based on program exchange rate of RF 553.7/US $\$$ and RF 549.9/US\$ for 2007.

${ }^{2}$ As a percent of the beginning-of-period stock of broad money.

${ }^{3}$ Increase in 2007 reflects rebasing of the monetary program; reserve money growth will be limited to 13 percent after correcting for the rebasing at end-2006.

${ }^{4}$ Revenue excluding grants minus current expenditure except interest due and exceptional expenditure minus domestically financed capital expenditure.

${ }^{5}$ Revenue excluding grants minus current expenditure (excluding external interest) minus domestically financed capital expenditure and net lending.

${ }^{6}$ After rescheduling, including arrears and new debt (the latter includes assumed project and budgetary disbursements for 2006-07)

and based on assumptions about expected new borrowing.

\section{CInternational Monetary Fund. Not for Redistribution}


Table 2. Rwanda: Operations of the Central Government, 2005-07

\begin{tabular}{|c|c|c|c|c|c|c|c|c|c|c|c|c|c|}
\hline & \multirow{3}{*}{$\begin{array}{l}2005 \\
\text { Actual }\end{array}$} & \multicolumn{4}{|c|}{2006} & \multicolumn{8}{|c|}{2007} \\
\hline & & \multirow{2}{*}{$\frac{\text { Jun }}{\text { Actual }}$} & \multirow{2}{*}{$\frac{\text { Sept }}{\text { Actual }}$} & \multicolumn{2}{|c|}{ Dec } & \multicolumn{2}{|c|}{ Mar } & \multicolumn{2}{|c|}{ Jun } & \multicolumn{2}{|c|}{ Sept } & \multicolumn{2}{|c|}{ Dec } \\
\hline & & & & $\begin{array}{c}\text { Country } \\
\text { Report } \\
\text { No. } 07 / 80\end{array}$ & Prel. & $\begin{array}{c}\text { Country } \\
\text { Reportt } \\
\text { No. } 07 / 80\end{array}$ & Proj. & $\begin{array}{c}\text { Country } \\
\text { Report } \\
\text { No. } 07 / 80\end{array}$ & Proj. & $\begin{array}{c}\text { Country } \\
\text { Report } \\
\text { No. } 07 / 80\end{array}$ & Proj. & $\begin{array}{c}\text { Country } \\
\text { Report } \\
\text { No. } 07 / 80\end{array}$ & Proj. \\
\hline & \multicolumn{13}{|c|}{ (Billions of Rwanda francs) } \\
\hline Revenue and grants & 349.4 & 225.4 & 301.2 & 374.3 & 376.0 & 103.6 & 74.5 & 244.2 & 227.9 & 322.3 & 335.5 & 398.3 & 433.2 \\
\hline $\begin{array}{l}\text { Total revenue } \\
\text { Of which: revenue measures }\end{array}$ & 180.3 & 99.3 & 151.6 & 197.9 & 208.2 & 48.7 & 56.7 & 104.8 & 109.4 & 159.2 & 166.1 & 217.1 & 226.6 \\
\hline Tax revenue & 162.6 & 92.3 & 141.6 & 186.1 & 193.6 & 45.6 & 53.6 & 99.6 & 103.1 & 151.4 & 156.4 & 207.4 & 214.5 \\
\hline Direct taxes & 50.7 & 34.4 & 47.1 & 59.8 & 64.5 & 15.3 & 18.8 & 33.5 & 34.5 & 50.9 & 52.5 & 69.7 & 72.0 \\
\hline Taxes on goods and services & 82.9 & 43.2 & 69.8 & 91.8 & 95.5 & 23.1 & 27.8 & 50.5 & 51.9 & 76.8 & 78.3 & 105.2 & 108.2 \\
\hline Taxes on international trade & 29.0 & 14.8 & 24.7 & 34.5 & 33.6 & 7.1 & 7.0 & 15.6 & 16.7 & 23.7 & 25.5 & 32.5 & 34.3 \\
\hline Nontax revenue & 17.7 & 6.9 & 10.0 & 11.8 & 14.6 & 3.1 & 3.1 & 5.3 & 6.3 & 7.8 & 9.8 & 9.7 & 12.1 \\
\hline Grants & 169.1 & 126.1 & 149.6 & 176.4 & 167.8 & 54.9 & 17.8 & 139.4 & 118.5 & 163.1 & 169.4 & 181.2 & 206.6 \\
\hline Budgetary grants & 111.3 & 58.0 & 67.6 & 80.5 & 71.9 & 39.9 & 5.0 & 109.5 & 93.0 & 117.2 & 130.2 & 123.5 & 144.9 \\
\hline Of which: HIPC Initiative assistance & 17.7 & 7.9 & 11.8 & 13.6 & 12.3 & 0.9 & 0.4 & 1.9 & 2.1 & 2.8 & 2.7 & 4.7 & 4.8 \\
\hline Capital grants & 57.8 & 68.1 & 82.0 & 95.9 & 95.9 & 15.0 & 12.8 & 29.9 & 25.6 & 45.9 & 39.2 & 57.7 & 61.7 \\
\hline Project grants & 57.8 & 25.7 & 39.6 & 53.5 & 53.5 & 15.0 & 12.8 & 29.9 & 25.6 & 45.9 & 39.2 & 57.7 & 61.7 \\
\hline MDRI grants & -- & 42.4 & 42.4 & 42.4 & 42.4 & 0.0 & 0.0 & 0.0 & 0.0 & 0.0 & 0.0 & \multicolumn{2}{|c|}{$\begin{array}{rr}0.0 & 0.0 \\
& 21.43703\end{array}$} \\
\hline Total expenditure and net lending & 340.7 & 181.6 & 288.1 & 376.8 & 382.5 & 97.6 & 105.0 & 204.8 & 219.9 & 313.2 & 337.7 & 4292 & $\begin{array}{r}21.43703 \\
464.5\end{array}$ \\
\hline Current expenditure & 214.9 & 125.2 & 193.5 & 253.7 & 254.1 & 63.5 & 67.3 & 132.7 & 142.6 & 204.4 & 222.4 & 280.4 & 304.7 \\
\hline Of which: priority & 99.5 & 61.3 & 99.2 & 126.2 & 127.7 & 31.8 & 32.6 & 70.0 & 76.7 & 108.0 & 121.7 & 145.1 & 165.5 \\
\hline Wages and salaries & 51.2 & 27.9 & 44.6 & 61.7 & 62.2 & 17.1 & 17.6 & 34.3 & 34.3 & 51.4 & 51.4 & 68.5 & 68.6 \\
\hline Civil & 34.7 & 19.5 & 30.2 & 40.7 & 41.2 & 10.9 & 11.3 & 24.0 & 24.0 & 35.1 & 35.1 & 47.5 & 47.5 \\
\hline Defense & 16.5 & 8.4 & 14.4 & 21.0 & 21.0 & 6.2 & 6.3 & 10.3 & 10.3 & 16.3 & 16.4 & 21.0 & 21.1 \\
\hline Purchases of goods and services & 64.5 & 36.2 & 57.2 & 70.0 & 71.6 & 15.0 & 17.3 & 30.9 & 32.0 & 47.5 & 51.3 & 64.5 & 71.5 \\
\hline Civil & 55.8 & 30.7 & 50.0 & 60.6 & 62.4 & 11.8 & 14.1 & 25.7 & 26.7 & 39.2 & 43.0 & 53.8 & 60.8 \\
\hline Defense & 8.6 & 5.5 & 7.1 & 9.4 & 9.1 & 3.2 & 3.2 & 5.2 & 5.2 & 8.3 & 8.3 & 10.7 & 10.7 \\
\hline Interest payments & 10.3 & 5.2 & 10.1 & 16.4 & 14.8 & 1.0 & 1.1 & 2.4 & 2.5 & 4.9 & 5.0 & 9.9 & 9.9 \\
\hline Domestic debt (due) & 4.2 & 1.4 & 4.7 & 10.4 & 8.6 & 0.6 & 0.7 & 1.6 & 1.7 & 3.6 & 3.6 & 8.1 & 8.1 \\
\hline External debt (due) & 6.1 & 3.8 & 5.4 & 6.0 & 6.2 & 0.5 & 0.4 & 0.8 & 0.8 & 1.3 & 1.4 & 1.8 & 1.8 \\
\hline Transfers & 53.5 & 35.8 & 54.4 & 72.5 & 71.9 & 21.0 & 20.3 & 45.1 & 50.6 & 69.6 & 79.5 & 95.0 & 107.4 \\
\hline Exceptional expenditure & 35.4 & 20.1 & 27.3 & 33.1 & 33.7 & 9.4 & 11.0 & 20.0 & 23.2 & 31.0 & 35.1 & 42.5 & 47.3 \\
\hline Of which: FARG & 7.8 & 4.5 & 6.7 & 9.2 & 9.0 & 2.6 & 2.8 & 5.2 & 5.5 & 7.7 & 8.3 & 10.3 & 11.1 \\
\hline Demobilization & 7.5 & 1.2 & 2.2 & 6.1 & 3.4 & 1.1 & 1.1 & 1.9 & 1.9 & 3.3 & 3.3 & 4.7 & 4.6 \\
\hline African Union peacekeeping & 9.2 & 8.2 & 9.7 & 10.4 & 10.9 & 3.1 & 3.1 & 5.1 & 5.1 & 8.1 & 8.1 & 10.7 & 10.7 \\
\hline Capital expenditure & 121.4 & 54.7 & 87.4 & 111.4 & 118.7 & 32.4 & 34.1 & 66.2 & 67.9 & 101.8 & 107.3 & 139.4 & 149.4 \\
\hline Of which: priority & 20.8 & 7.0 & 11.1 & 14.2 & 19.8 & 6.8 & 7.0 & 15.2 & 16.0 & 22.9 & 28.5 & 32.9 & 41.5 \\
\hline Domestic & 34.7 & 14.9 & 26.4 & 28.4 & 35.7 & 9.4 & 11.0 & 20.0 & 21.8 & 31.1 & 36.6 & 43.2 & 53.2 \\
\hline Foreign & 86.7 & 39.8 & 61.0 & 83.0 & 83.0 & 23.1 & 23.1 & 46.1 & 46.1 & 70.7 & 70.7 & 96.2 & 96.2 \\
\hline Net lending ${ }^{1}$ & 4.4 & 1.7 & 7.2 & 11.7 & 9.6 & 1.6 & 3.6 & 6.0 & 9.4 & 7.0 & 8.0 & 9.4 & 10.4 \\
\hline Of which: priority & 0.0 & 0.0 & 3.7 & 3.7 & 4.1 & 0.0 & 0.0 & 1.5 & 1.5 & 1.5 & 1.5 & 3.2 & 3.2 \\
\hline Primary balance $^{2}$ & -23.6 & -20.7 & -31.8 & -39.4 & -31.2 & -13.8 & -9.4 & -25.4 & -30.7 & -40.3 & -51.2 & -53.4 & -68.2 \\
\hline Domestic fiscal balance $^{3}$ & -67.6 & -38.7 & -70.1 & -89.9 & -85.1 & -25.3 & -24.8 & -53.0 & -63.6 & -82.0 & -99.5 & -114.1 & -139.9 \\
\hline Excluding demobilization and peacekeeping expenditures ${ }^{4}$ & -50.9 & -29.3 & -58.2 & -73.3 & -70.8 & -21.1 & -20.6 & -46.0 & -56.6 & -70.6 & -88.1 & -98.7 & -124.6 \\
\hline Overall deficit ( & & & & & & & & & & & & & \\
\hline After grants & 9.0 & 43.7 & 13.1 & -2.5 & -6.5 & 6.1 & -30.4 & 39.4 & 8.0 & 9.1 & -2.1 & -30.9 & -31.2 \\
\hline Excluding MDRI grants & -- & 1.3 & -29.3 & -44.9 & -48.9 & -- & -- & -- & -- & -- & -- & -- & \\
\hline Before grants & -160.4 & -82.4 & -136.5 & -178.9 & -174.3 & -48.8 & -48.2 & -100.0 & -110.5 & -154.0 & -171.5 & -212.1 & -237.9 \\
\hline Change in arrears ${ }^{5}$ & -7.0 & -4.4 & -4.6 & -7.0 & -7.5 & -1.6 & -1.6 & -3.9 & -3.9 & -5.4 & -5.4 & -7.0 & -7.0 \\
\hline Domestic & -7.0 & -4.4 & -4.6 & -7.0 & -7.5 & -1.6 & -1.6 & -3.9 & -3.9 & -5.4 & -5.4 & -7.0 & -7.0 \\
\hline External & 0.0 & 0.0 & 0.0 & 0.0 & 0.0 & 0.0 & 0.0 & 0.0 & 0.0 & 0.0 & 0.0 & 0.0 & 0.0 \\
\hline Deficit (-) & 2.0 & 39.3 & 8.5 & -9.5 & -14.0 & 4.5 & -32.0 & 35.5 & 4.1 & 3.7 & -7.5 & -37.9 & -38.2 \\
\hline
\end{tabular}


Table 2. Rwanda: Operations of the Central Government, 2005-07 (concluded)

\begin{tabular}{|c|c|c|c|c|c|c|c|c|c|c|c|c|c|}
\hline & \multirow{3}{*}{$\begin{array}{l}2005 \\
\text { Actual }\end{array}$} & \multicolumn{4}{|c|}{2006} & \multicolumn{8}{|c|}{2007} \\
\hline & & \multirow{2}{*}{$\begin{array}{c}\text { Jun } \\
\text { Actual }\end{array}$} & \multirow{2}{*}{$\begin{array}{l}\text { Sept } \\
\text { Prel. }\end{array}$} & \multicolumn{2}{|c|}{ Dec } & \multicolumn{2}{|c|}{ Mar } & \multicolumn{2}{|c|}{ Jun } & \multicolumn{2}{|c|}{ Sept } & \multicolumn{2}{|c|}{ Dec } \\
\hline & & & & $\begin{array}{c}\text { Country } \\
\text { Report } \\
\text { No. } 07 / 80\end{array}$ & Prel. & $\begin{array}{c}\text { Country } \\
\text { Report } \\
\text { No. } 07 / 80\end{array}$ & Proj. & $\begin{array}{c}\text { Country } \\
\text { Report } \\
\text { No. } 07 / 80\end{array}$ & Proj. & $\begin{array}{c}\text { Country } \\
\text { Report } \\
\text { No. } 07 / 80\end{array}$ & Proj. & $\begin{array}{c}\text { Country } \\
\text { Report } \\
\text { No. } 07 / 80\end{array}$ & Proj. \\
\hline Financing & -9.0 & -44.8 & -14.4 & 9.5 & 15.2 & -4.5 & 32.0 & -35.5 & -4.1 & -3.7 & 7.5 & 37.9 & 38.2 \\
\hline Foreign financing (net) & 30.9 & 6.0 & 13.9 & 20.2 & 25.9 & 7.0 & 9.8 & 14.3 & 19.6 & 22.0 & 29.9 & 33.1 & 31.0 \\
\hline Drawings & 46.6 & 14.6 & 25.2 & 33.3 & 37.7 & 8.1 & 10.6 & 16.2 & 21.5 & 24.8 & 32.7 & 38.5 & 36.4 \\
\hline Budgetary loans & 17.7 & 0.5 & 3.8 & 3.8 & 8.2 & 0.0 & 0.3 & 0.0 & 0.9 & 0.0 & 1.3 & 0.0 & 1.9 \\
\hline Project loans & 28.8 & 14.1 & 21.4 & 29.5 & 29.5 & 8.1 & 10.3 & 16.2 & 20.6 & 24.8 & 31.5 & 38.5 & 34.5 \\
\hline Amortization & -21.3 & -8.6 & -11.3 & -13.1 & -11.8 & -1.1 & -0.7 & -1.9 & -1.9 & -2.8 & -2.8 & -5.3 & -5.4 \\
\hline Exceptional financing & 5.6 & 0.0 & 0.0 & 0.0 & 0.0 & 0.0 & 0.0 & 0.0 & 0.0 & 0.0 & 0.0 & 0.0 & 0.0 \\
\hline Net domestic financing & -39.9 & -50.8 & -28.2 & -10.7 & -10.7 & -11.5 & 22.2 & -49.8 & -23.7 & -25.7 & -22.4 & 4.7 & 7.2 \\
\hline Banking system treasury operations (monetary survey) & -16.8 & -45.0 & -27.2 & -8.8 & -11.2 & -9.9 & 17.4 & -46.0 & -26.7 & -21.9 & -24.1 & 10.1 & 0.4 \\
\hline Nonbank sector (including CSR repayment) ${ }^{6}$ & -15.7 & -5.8 & -1.0 & -1.9 & 0.5 & -1.6 & 4.8 & -3.8 & 3.0 & -3.8 & 1.7 & -5.4 & 6.8 \\
\hline Errors and omissions ${ }^{7}$ & -7.0 & -5.5 & -5.9 & 0.0 & 1.2 & 0.0 & 0.0 & 0.0 & 0.0 & 0.0 & 0.0 & 0.0 & 0.0 \\
\hline Financing gap (+) & 0.0 & 0.0 & 0.0 & 0.0 & 0.0 & 0.0 & 0.0 & 0.0 & 0.0 & 0.0 & 0.0 & 0.0 & 0.0 \\
\hline Memorandum items: & \multicolumn{13}{|c|}{ (Percent of GDP, unless otherwise indicated) } \\
\hline Revenue and grants & 29.2 & 16.4 & 21.9 & 28.4 & 27.3 & 7.2 & 4.8 & 16.9 & 14.8 & 22.3 & 21.8 & 27.6 & 28.1 \\
\hline Revenue, excluding grants & 15.1 & 7.2 & 11.0 & 15.0 & 15.1 & 3.4 & 3.7 & 7.3 & 7.1 & 11.0 & 10.8 & 15.0 & 14.7 \\
\hline ts (percent of monetized sector GDP) & 29.5 & 14.0 & 21.3 & 28.9 & 29.3 & 6.5 & 7.0 & 13.9 & 13.5 & 21.2 & 20.6 & 28.9 & 28.1 \\
\hline Total expenditure & 28.5 & 13.1 & 20.9 & 28.6 & 27.8 & 6.8 & 6.8 & 14.2 & 14.3 & 21.7 & 21.9 & 29.7 & 30.1 \\
\hline Of which: total priority & 10.0 & 5.0 & 8.3 & 10.9 & 11.0 & 2.7 & 2.6 & 6.0 & 6.1 & 9.2 & 9.8 & 12.5 & 13.6 \\
\hline Current expenditure & 17.9 & 9.0 & 14.1 & 19.3 & 18.5 & 4.4 & 4.4 & 9.2 & 9.3 & 14.1 & 14.4 & 19.4 & 19.8 \\
\hline Of which: wage bill & 4.3 & 2.0 & 3.2 & 4.7 & 4.5 & 1.2 & 1.1 & 2.4 & 2.2 & 3.6 & 3.3 & 4.7 & 4.5 \\
\hline goods and services & 5.4 & 2.6 & 4.2 & 5.3 & 5.2 & 1.0 & 1.1 & 2.1 & 2.1 & 3.3 & 3.3 & 4.5 & 4.6 \\
\hline Of which: defense/security & 2.1 & 1.0 & 1.6 & 2.3 & 2.2 & 0.7 & 0.6 & 1.1 & 1.0 & 1.7 & 1.6 & 2.2 & 2.1 \\
\hline exceptional expenditure & 3.0 & 1.5 & 2.0 & 2.5 & 2.4 & 0.6 & 0.7 & 1.4 & 1.5 & 2.1 & 2.3 & 2.9 & 3.1 \\
\hline Capital expenditure & 10.1 & 4.0 & 6.4 & 8.5 & 8.6 & 2.2 & 2.2 & 4.6 & 4.4 & 7.0 & 7.0 & 9.6 & 9.7 \\
\hline Domestic fiscal balance & -5.6 & -2.8 & -5.1 & -6.8 & -6.2 & -1.8 & -1.6 & -3.7 & -4.1 & -5.7 & -6.5 & -7.9 & -9.1 \\
\hline Excluding demobilization and peacekeeping expenditures ${ }^{4}$ & -4.3 & -2.1 & -4.2 & -5.6 & -5.1 & -1.5 & -1.3 & -3.2 & -3.7 & -4.9 & -5.7 & -6.8 & -8.1 \\
\hline Of which: domestic expenditure & 19.3 & 9.3 & 15.3 & 20.6 & 20.3 & 4.8 & 5.0 & 10.4 & 10.8 & 15.9 & 16.5 & 21.9 & 22.8 \\
\hline Primary balance $^{2}$ & -2.0 & -1.5 & -2.3 & -3.0 & -2.3 & -1.0 & -0.6 & -1.8 & -2.0 & -2.8 & -3.3 & -3.7 & -4.8 \\
\hline Overall deficit (payment order) & & & & & & & & & & & & & \\
\hline After grants & 0.7 & 3.2 & 1.0 & -0.2 & -0.5 & 0.4 & -2.0 & 2.7 & 0.5 & 0.6 & -0.1 & -2.1 & -2.0 \\
\hline Before grants & -13.4 & -6.0 & -9.9 & -13.6 & -12.7 & -3.4 & -3.1 & -6.9 & -7.2 & -10.7 & -11.1 & -14.7 & -15.4 \\
\hline $\begin{array}{l}\text { Before grants (percent of monetized sector GDP) } \\
\text { ) }\end{array}$ & -26.2 & $\begin{array}{r}-11.6 \\
1375 .\end{array}$ & -19.2 & -26.1 & $\begin{array}{r}-24.5 \\
13757\end{array}$ & $\begin{array}{r}-6.5 \\
-1445\end{array}$ & -6.0 & -13.3 & -13.7 & -20.5 & -21.3 & -28.2 & -29.5 \\
\hline Nominal GDP (RF billions) & $1,197.2$ & $1,375.7$ & $1,375.7$ & $1,316.1$ & $1,375.7$ & $1,444.5$ & $1,540.6$ & $1,444.5$ & $1,540.6$ & $1,444.5$ & $1,540.6$ & $1,444.5$ & $1,540.6$ \\
\hline Net credit to government from banking system (RF billions) & -30.8 & -46.4 & -25.9 & -8.8 & -7.8 & -6.7 & 21.2 & -40.5 & -21.1 & -8.8 & -10.8 & 25.1 & 15.4 \\
\hline Of which: treasury operations & -16.8 & -45.0 & -27.2 & -8.8 & -11.2 & -9.9 & 17.4 & -46.0 & -26.7 & -21.9 & -24.1 & 10.1 & 0.4 \\
\hline project accou & -14.0 & -1.4 & 1.3 & 0.0 & 3.4 & 3.2 & 3.8 & 5.5 & 5.6 & 13.1 & 13.3 & 15.0 & 15.0 \\
\hline other & 0.0 & 0.0 & 0.0 & 0.0 & 0.0 & 0.0 & 0.0 & 0.0 & 0.0 & 0.0 & 0.0 & 0.0 & 0.0 \\
\hline MDRI stock adjustment ( & $\ldots$ & 42.4 & 673.5 & 673.5 & 677.2 & & & & & & & & \\
\hline MDRI debt service savings & $\ldots$ & 0.3 & 0.4 & 0.5 & 0.5 & 0.1 & 0.1 & 0.3 & 0.2 & 0.4 & 0.4 & 0.5 & 0.5 \\
\hline MDRI debt service savings from AfDB (RF billions) & $\ldots$ & & 1.8 & 1.8 & 1.8 & 0.5 & 0.3 & 0.9 & 0.9 & 1.4 & 1.4 & 1.8 & 1.8 \\
\hline MDRI debt service savings from IMF (RF billions) & & 4.1 & 4.1 & 4.1 & 4.1 & 1.1 & 0.8 & 2.2 & 2.2 & 3.2 & 3.3 & 4.3 & 4.4 \\
\hline MDRI debt service savings from IDA ( $R F$ billions) & & & & 1.3 & 1.3 & 0.3 & 0.3 & 0.7 & 0.7 & 1.0 & 1.0 & 1.4 & 1.4 \\
\hline
\end{tabular}

Sources: Rwandese authorities, and IMF staff estimates and projections.

${ }^{1}$ Net lending in 2005 incorporates RF2.9 billion in receipts from the privatization of Rwandatel.

${ }^{2}$ Definition excludes exceptional expenditures, defined as total revenue (excluding privatization proceeds) minus noninterest current expenditure (excluding exceptional expenditure) minus domestically financed capital expenditure.

${ }^{3}$ Revenue excluding grants minus current expenditure, domestically financed capital expenditure, and net lending, excluding external interest.

${ }^{4}$ Actual data for 2005 exclude Rf8.5 billion expenditures for peacekeeping operations, which were not covered by grants. The domestic deficit excluding grant-financed peacekeeping would have been 5 percent of GDP in 2005. For 2006, peacekeeping activities of Rf 2.9 billion were not covered by grants.

A negative sign indicates a reduction.
${ }^{6} \mathrm{CSR}=$ Caisse Sociale du Rwanda.

A negative number implies a discrepancy that is consistent with an underestimate of financing. 
Table 3. Rwanda: Monetary Survey, 2005-07

\begin{tabular}{|c|c|c|c|c|c|c|c|c|c|c|c|c|}
\hline & \multirow{3}{*}{$\begin{array}{l}2005 \\
\text { Act }^{1}\end{array}$} & \multicolumn{4}{|c|}{2006} & \multicolumn{7}{|c|}{2007} \\
\hline & & \multirow{2}{*}{$\frac{\text { Jun }}{\text { Act }^{1}}$} & \multirow{2}{*}{$\frac{\text { Sep. }}{\text { Act }^{1}}$} & \multicolumn{2}{|c|}{ Dec } & \multirow{2}{*}{$\begin{array}{l}\text { Mar } \\
\text { Proj }\end{array}$} & \multicolumn{2}{|c|}{ Jun } & \multicolumn{2}{|c|}{ Sept } & \multicolumn{2}{|c|}{ Dec } \\
\hline & & & & $\begin{array}{c}\text { Country } \\
\text { Report } \\
\text { No. } 07 / 80\end{array}$ & Act. $^{1}$ & & $\begin{array}{c}\text { Country } \\
\text { Report } \\
\text { No. } 07 / 80\end{array}$ & Proj & $\begin{array}{c}\text { Country } \\
\text { Report } \\
\text { No. } 07 / 80\end{array}$ & Proj & $\begin{array}{c}\text { Country } \\
\text { Report } \\
\text { No. } 07 / 80\end{array}$ & Proj. ${ }^{1}$ \\
\hline Monetary authorities & \multicolumn{12}{|c|}{ (RF billions) } \\
\hline Net foreign assets & 185.6 & 236.6 & 228.0 & 200.9 & 227.4 & 208.6 & 244.3 & 251.4 & 206.8 & 245.7 & 179.3 & 217.7 \\
\hline Foreign assets & 230.0 & 240.3 & 231.5 & 204.2 & 243.9 & 225.5 & 248.7 & 255.8 & 211.2 & 250.1 & 184.9 & 223.3 \\
\hline Foreign liabilities ${ }^{2}$ & 44.4 & 3.7 & 3.5 & 3.3 & 16.4 & 16.9 & 4.4 & 4.4 & 4.4 & 4.4 & 5.6 & 5.6 \\
\hline Net domestic assets & -121.4 & -167.0 & -157.3 & -128.4 & -155.6 & -126.1 & -164.8 & -163.5 & -126.3 & -156.2 & -97.4 & -125.4 \\
\hline Domestic credit & -96.7 & -136.1 & -119.5 & -96.4 & -120.2 & -94.1 & -132.8 & -131.5 & -94.3 & -124.2 & -65.4 & -93.4 \\
\hline Government (net) & -60.5 & -98.3 & -77.1 & -69.9 & -72.4 & -51.1 & -110.4 & -93.5 & -78.7 & -83.2 & -44.8 & -57.0 \\
\hline Claims & 42.1 & 41.8 & 41.8 & 42.1 & 41.8 & 41.8 & 42.1 & 41.8 & 42.1 & 41.8 & 42.1 & 41.8 \\
\hline Of which: overdraft & -- & -- & -- & -- & -- & -- & -- & -- & -- & -- & -- & - \\
\hline Deposits (excluding autonomous bodies) ${ }^{2}$ & 102.6 & 140.1 & 118.9 & 112.0 & 114.2 & 92.9 & 152.6 & 135.3 & 120.9 & 125.0 & 86.9 & 98.8 \\
\hline Public nongovernment deposits (-) & -0.8 & -0.5 & -0.9 & -0.8 & -2.0 & -0.9 & -0.8 & -2.0 & -0.9 & -0.9 & -0.8 & -2.0 \\
\hline Nongovernment credit & -35.3 & -37.3 & -41.5 & -25.8 & -45.8 & -42.0 & -21.6 & -36.0 & -14.6 & -40.1 & -19.9 & -34.4 \\
\hline Private sector & 3.4 & 3.4 & 3.5 & 3.4 & 3.5 & 3.5 & 3.4 & 3.5 & 3.5 & 3.5 & 3.4 & 3.5 \\
\hline Public enterprises & 0.1 & -- & -- & 0.1 & -- & -- & 0.1 & -- & 0.1 & -- & 0.1 & 0.1 \\
\hline Commercial banks & -38.9 & -40.7 & -45.0 & -29.3 & -49.3 & -45.5 & -25.2 & -39.6 & -18.2 & -43.6 & -23.4 & -38.0 \\
\hline Discount window & 1.4 & 1.3 & 1.2 & 1.4 & 1.2 & -0.6 & 0.4 & 0.4 & 1.4 & 1.4 & 1.4 & 1.2 \\
\hline Money market (- = absorption) & -40.2 & -42.0 & -46.2 & -30.7 & -50.5 & -44.9 & -25.6 & -40.0 & -19.6 & -45.0 & -24.8 & -39.2 \\
\hline Other items (net; asset +) & -24.7 & -30.9 & -37.7 & -32.0 & -35.4 & -32.0 & -32.0 & -32.0 & -32.0 & -32.0 & -32.0 & -32.0 \\
\hline Reserve money ${ }^{3 / 4}$ & 64.2 & 69.6 & 70.7 & 72.4 & 71.8 & 82.6 & 79.5 & 87.9 & 80.5 & 89.4 & 81.9 & 92.4 \\
\hline Currency in circulation & 48.3 & 51.2 & 48.0 & 52.7 & 54.6 & 52.0 & 56.5 & 56.5 & 58.2 & 58.2 & 58.5 & 60.6 \\
\hline Commercial bank reserves & 13.7 & 16.1 & 19.7 & 17.5 & 15.1 & 28.5 & 20.8 & 29.3 & 20.1 & 29.1 & 21.1 & 29.6 \\
\hline Nonbank deposits & 2.2 & 2.2 & 3.0 & 2.2 & 2.1 & 2.1 & 2.2 & 2.1 & 2.2 & 2.1 & 2.2 & 2.1 \\
\hline Of which: autonomous public agencies & 0.4 & 0.3 & 0.4 & -- & 0.4 & -2.0 & -1.0 & -1.0 & -- & -- & -- & -- \\
\hline \multicolumn{13}{|l|}{ Commercial banks } \\
\hline Net foreign assets & 42.1 & 42.9 & 54.0 & 42.0 & 60.8 & 47.0 & 44.0 & 49.0 & 49.0 & 46.0 & 43.5 & 48.7 \\
\hline Foreign assets & 53.8 & 54.8 & 66.3 & 54.0 & 71.7 & 57.9 & 56.0 & 59.9 & 61.0 & 56.9 & 55.5 & 59.6 \\
\hline Foreign liabilities & 11.7 & 12.0 & 12.3 & 12.0 & 10.9 & 10.9 & 12.0 & 10.9 & 12.0 & 10.9 & 12.0 & 10.9 \\
\hline Reserves & 13.7 & 16.1 & 19.7 & 17.5 & 15.1 & 28.5 & 20.8 & 29.3 & 20.1 & 29.1 & 21.1 & 29.6 \\
\hline NBR deposits & 10.9 & 11.9 & 13.8 & 14.5 & 11.3 & 18.7 & 17.9 & 20.4 & 19.1 & 21.9 & 16.1 & 23.9 \\
\hline Required reserves & 14.9 & 17.2 & 18.2 & 17.4 & 18.8 & 20.2 & 18.4 & 20.9 & 18.6 & 21.4 & 19.1 & 21.8 \\
\hline Excess reserves & -4.0 & -5.4 & -4.4 & -3.0 & -7.5 & -1.5 & -0.5 & -0.5 & 0.5 & 0.5 & -3.0 & 2.1 \\
\hline Cash in vault & 2.8 & 4.2 & 5.9 & 3.0 & 3.8 & 3.8 & 3.0 & 3.8 & 3.0 & 3.8 & 5.0 & 5.8 \\
\hline Net credit from NBR (rediscount; liability -) & 38.9 & 40.7 & 45.0 & 29.3 & 49.3 & 45.5 & 25.2 & 39.6 & 18.2 & 43.6 & 23.4 & 38.0 \\
\hline Domestic credit & 128.7 & 138.8 & 145.3 & 159.6 & 164.0 & 166.8 & 173.7 & 179.7 & 178.8 & 184.7 & 184.3 & 191.7 \\
\hline Government (net) & -1.1 & -9.7 & -10.4 & -0.5 & 3.0 & 3.0 & -0.5 & 3.0 & -0.5 & 3.0 & -0.5 & 3.0 \\
\hline Credit & 21.1 & 12.8 & 12.8 & 21.1 & 25.6 & 25.6 & 21.1 & 25.6 & 21.1 & 25.6 & 21.1 & 25.6 \\
\hline Deposits & 22.2 & 22.5 & 23.1 & 21.6 & 22.6 & 22.6 & 21.6 & 22.6 & 21.6 & 22.6 & 21.6 & 22.6 \\
\hline Public enterprises & 2.1 & 2.3 & 1.4 & 2.1 & 2.4 & 2.4 & 2.1 & 2.4 & 2.1 & 2.4 & 2.1 & 2.4 \\
\hline Private sector & 127.6 & 146.2 & 154.3 & 158.0 & 158.7 & 161.5 & 172.0 & 174.3 & 177.1 & 179.4 & 182.7 & 186.4 \\
\hline Other items (net; asset +) & -54.3 & -50.7 & -57.0 & -52.0 & -58.4 & -58.4 & -55.0 & -58.4 & -55.0 & -58.4 & -55.0 & -58.4 \\
\hline Deposits & 169.1 & 187.7 & 207.0 & 196.4 & 230.8 & 229.4 & 208.6 & 239.1 & 211.0 & 245.0 & 217.4 & 249.7 \\
\hline Private & 136.8 & 152.8 & 167.4 & 164.2 & 186.4 & 185.0 & 176.4 & 194.7 & 178.8 & 200.6 & 185.2 & 205.3 \\
\hline Public (nongovernment) & 32.2 & 34.9 & 39.6 & 32.2 & 44.4 & 44.4 & 32.2 & 44.4 & 32.2 & 44.4 & 32.2 & 44.4 \\
\hline
\end{tabular}


Table 3. Rwanda: Monetary Survey, 2005-07 (concluded)

\begin{tabular}{|c|c|c|c|c|c|c|c|c|c|c|c|c|}
\hline & \multirow{3}{*}{$\begin{array}{l}2005 \\
\text { Act }^{1}\end{array}$} & \multicolumn{4}{|c|}{2006} & \multicolumn{7}{|c|}{2007} \\
\hline & & \multirow{2}{*}{$\frac{\text { Jun }}{\text { Act }^{1}}$} & \multirow{2}{*}{$\frac{\text { Sep. }}{\text { Act }^{\prime}}$} & \multicolumn{2}{|c|}{ Dec } & \multirow{2}{*}{$\begin{array}{l}\text { Mar } \\
\text { Proj }\end{array}$} & \multicolumn{2}{|c|}{ Jun } & \multicolumn{2}{|c|}{ Sept } & \multicolumn{2}{|c|}{ Dec } \\
\hline & & & & Proj. $^{1}$ & Act. $^{\top}$ & & $\begin{array}{c}\text { Country } \\
\text { Report } \\
\text { No. } 07 / 80\end{array}$ & Proj & $\begin{array}{c}\text { Country } \\
\text { Report } \\
\text { No. } 07 / 80\end{array}$ & Proj & $\begin{array}{c}\text { Country } \\
\text { Report } \\
\text { No. } 07 / 80\end{array}$ & Proj. ${ }^{1}$ \\
\hline Monetary survey & \multicolumn{12}{|c|}{ (RF billions) } \\
\hline Net foreign assets & 227.7 & 279.4 & 282.0 & 242.9 & 288.2 & 255.6 & 288.3 & 300.4 & 255.8 & 291.7 & 222.8 & 266.4 \\
\hline Net domestic assets & -8.2 & -38.2 & -24.0 & 8.5 & -0.7 & 27.9 & -21.0 & -2.6 & 15.7 & 13.7 & 55.3 & 46.0 \\
\hline Domestic credit & 70.9 & 43.4 & 70.8 & 92.5 & 93.2 & 118.3 & 66.0 & 87.8 & 102.7 & 104.1 & 142.3 & 136.4 \\
\hline Government (net) & -61.6 & -108.0 & -87.5 & -70.4 & -69.4 & -48.2 & -110.9 & -90.5 & -79.2 & -80.3 & -45.3 & -54.0 \\
\hline Public nongovernment deposits (-) & -0.8 & -0.5 & -0.9 & -0.8 & -2.0 & -0.9 & -0.8 & -2.0 & -0.9 & -0.9 & -0.8 & -2.0 \\
\hline Public enterprises & 2.2 & 2.3 & 1.4 & 2.2 & 2.4 & 2.4 & 2.2 & 2.4 & 2.2 & 2.4 & 2.2 & 2.5 \\
\hline Private sector & 131.1 & 149.6 & 157.8 & 161.4 & 162.2 & 164.9 & 175.5 & 177.8 & 180.6 & 182.8 & 186.1 & 189.9 \\
\hline Other items (net; asset +) & -79.0 & -81.7 & -94.8 & -84.0 & -93.8 & -90.4 & -87.0 & -90.4 & -87.0 & -90.4 & -87.0 & -90.4 \\
\hline Broad money $^{5 / 6}$ & 219.6 & 241.2 & 258.0 & 251.4 & 287.6 & 283.5 & 267.3 & 297.7 & 271.5 & 305.3 & 278.1 & 312.4 \\
\hline Currency in circulation & 48.3 & 51.2 & 48.0 & 52.7 & 54.6 & 52.0 & 56.5 & 56.5 & 58.2 & 58.2 & 58.5 & 60.6 \\
\hline Deposits & 171.3 & 190.0 & 210.0 & 198.7 & 232.9 & 231.5 & 210.8 & 241.2 & 213.3 & 247.1 & 219.6 & 251.8 \\
\hline \multirow[t]{2}{*}{ Of which: foreign currency deposits } & 39.4 & 41.2 & 53.1 & 40.0 & 53.1 & 47.0 & 36.0 & 43.0 & 37.0 & 40.0 & 41.0 & 45.0 \\
\hline & \multicolumn{12}{|c|}{ (Annual changes in percent of beginning-of-year of broad money) } \\
\hline Net foreign assets & 29.3 & 49.8 & 54.0 & 6.9 & 29.9 & -2.5 & 4.5 & 9.5 & -9.4 & 4.5 & -8.0 & -7.4 \\
\hline Net domestic assets & -12.1 & -30.3 & -24.9 & 7.6 & 1.6 & 30.8 & 6.5 & 14.1 & 14.7 & 14.0 & 18.6 & 16.1 \\
\hline Domestic credit & -5.3 & -20.0 & -10.2 & 9.9 & 10.2 & 40.3 & 9.4 & 18.4 & 12.4 & 12.9 & 19.8 & 15.0 \\
\hline Government (net) & -16.4 & -32.9 & -25.7 & -4.0 & -3.6 & 28.4 & -1.2 & 7.3 & 3.2 & 2.8 & 10.0 & 5.4 \\
\hline Economy & 11.2 & 12.9 & 15.4 & 13.8 & 13.8 & 11.9 & 10.6 & 11.1 & 9.2 & 10.1 & 9.8 & 9.7 \\
\hline Other items (net; asset +) & -6.9 & -10.3 & -14.7 & -2.3 & -8.6 & -9.5 & -2.9 & -4.3 & 2.3 & 1.1 & -1.2 & 1.0 \\
\hline Broad money & 17.2 & 19.5 & 29.1 & 14.5 & 31.5 & 28.3 & 11.0 & 23.6 & 5.3 & 18.5 & 10.6 & 8.7 \\
\hline & \multicolumn{12}{|c|}{ (Annual percent changes) } \\
\hline Net foreign assets & 31.8 & 56.2 & 62.0 & 6.7 & 29.3 & -2.8 & 3.9 & 8.2 & -8.7 & 4.1 & -8.3 & -7.4 \\
\hline Net domestic assets & -156.0 & -296.3 & -205.7 & -204.1 & -92.0 & -166.4 & -45.1 & -93.1 & -165.8 & -157.0 & 550.9 & $-21,773.7$ \\
\hline Domestic credit & -12.2 & -48.1 & -22.4 & 30.5 & 31.5 & 305.7 & 52.0 & 102.1 & 45.0 & 47.0 & 53.9 & 46.4 \\
\hline Government (net) & 100.0 & 159.4 & 142.1 & 14.2 & 12.7 & -56.6 & 2.7 & -16.2 & -9.4 & -8.2 & -35.7 & -22.2 \\
\hline Economy & 18.8 & 20.8 & 24.2 & 22.9 & 22.8 & 18.8 & 16.8 & 17.7 & 14.9 & 16.5 & 15.2 & 17.1 \\
\hline Other items (net; asset +) & 19.5 & 34.3 & 45.0 & 6.3 & 25.1 & 27.1 & 8.7 & 13.0 & -6.5 & -3.0 & 3.6 & -3.2 \\
\hline & \multicolumn{12}{|c|}{ (Percent, unless otherwise indicated) } \\
\hline \multicolumn{13}{|l|}{ Memorandum items: } \\
\hline Currency/broad money ratio & 22.0 & 21.2 & 18.6 & 21.0 & 19.0 & 18.3 & 21.1 & 19.0 & 21.4 & 19.1 & 21.0 & 19.4 \\
\hline Reserve money annual growth & 12.3 & 14.9 & 16.2 & 12.8 & 11.9 & 27.0 & 14.3 & 26.4 & 13.8 & 26.4 & 13.0 & 28.5 \\
\hline Reserves/deposits & 38.0 & 37.1 & 34.2 & 36.9 & 31.1 & 36.0 & 38.1 & 36.8 & 38.2 & 36.5 & 37.7 & 37.0 \\
\hline Money multiplier & 3.4 & 3.5 & 3.6 & 3.5 & 4.0 & 3.4 & 3.4 & 3.4 & 3.4 & 3.4 & 3.4 & 3.4 \\
\hline Reserve/deposits & 8.0 & 7.8 & 8.3 & 8.8 & 6.1 & 10.1 & 10.2 & 10.5 & 10.7 & 10.9 & 8.7 & 11.6 \\
\hline Currency/deposits & 35.3 & 33.5 & 28.7 & 32.1 & 29.3 & 28.1 & 32.0 & 29.0 & 32.6 & 29.0 & 31.6 & 29.5 \\
\hline Velocity of broad money (end of period) & 5.5 & $\ldots$ & $\ldots$ & 5.2 & 4.8 & 4.9 & 5.2 & 4.9 & 5.2 & 4.9 & 5.2 & 4.9 \\
\hline Velocity of broad money (average of period) & 5.9 & $\ldots$ & $\ldots$ & 5.4 & 5.5 & 5.1 & 5.4 & 5.1 & 5.4 & 5.1 & 5.4 & 5.1 \\
\hline Net open position of the NBR (RF billion) & 181.2 & 232.0 & 222.6 & 197.7 & 221.6 & 204.9 & 241.1 & 247.6 & 203.6 & 241.9 & 176.0 & 214.1 \\
\hline Net open position of commercial banks (RF billion) & 7.2 & 1.0 & 5.0 & 2.0 & 1.0 & 0.0 & 8.0 & 6.0 & 12.0 & 6.0 & 2.5 & 3.7 \\
\hline Extended broad money (RF billion) ${ }^{\prime}$ & 250.9 & 283.1 & 303.2 & 287.3 & 334.4 & 329.6 & 305.6 & 346.2 & 310.3 & 355.0 & 317.9 & 363.3 \\
\hline Nominal GDP (RF billion) & $1,197.2$ & $\ldots$ & $\ldots$ & $1,316.1$ & $1,375.7$ & $\ldots$ & & $\ldots$ & $\ldots$ & $\ldots$ & $1,444.5$ & $1,540.6$ \\
\hline
\end{tabular}

${ }^{1}$ Converted at the program exchange rate: for 2005: RF 566.9/US\$; for 2006: RF 553.7/US\$; and for 2007: RF 549.9/US\$.

${ }^{2}$ The IMF's MDRI reduced foreign liabilities at the NBR by RF 42.4 million with a counter entry in government deposits (in January 2006).

${ }^{3}$ The definition of reserve money as performance criterion or structural benchmark differs from the definition in the monetary program in that it excludes the deposits of a defunct

savings bank, import deposits, and dormant accounts. It includes old notes demonetized at the end of 2004 but in circulation until the end of 2005.

${ }^{4}$ The reserve money target for 2007 is derived by applying the programmed growth rate of 13 percent to the rebased 2006 outcome. The rebased 2006 outcome was calculated by

allowing banks to meet the legal reserve requirement on deposits (banks' reserves at the end of December 2006 fell short of the requirement by RF 7.5 billion, they met the requirement

because it is based on an average over two weeks; plus (2) a liquidity reserve of RF 3 billion to ensure sufficient financing in banks' branches.

${ }^{5}$ From December 2005 on, includes Caisse Hypothecaire du Rwanda (BHR) deposits (RF 1 billion at end-2005).

${ }^{6}$ End-2006 broad money includes RF5 billion temporary build up of local government deposits, which were unwound by February 2007.

${ }^{7}$ Broad money plus deposits in the Union de Banques Populaires de Rwanda (UBPR) and Rwanda Development Bank (BRD). 
Table 4. Rwanda: Balance of Payments, 2003-09 (millions US\$, unless otherwise indicated)

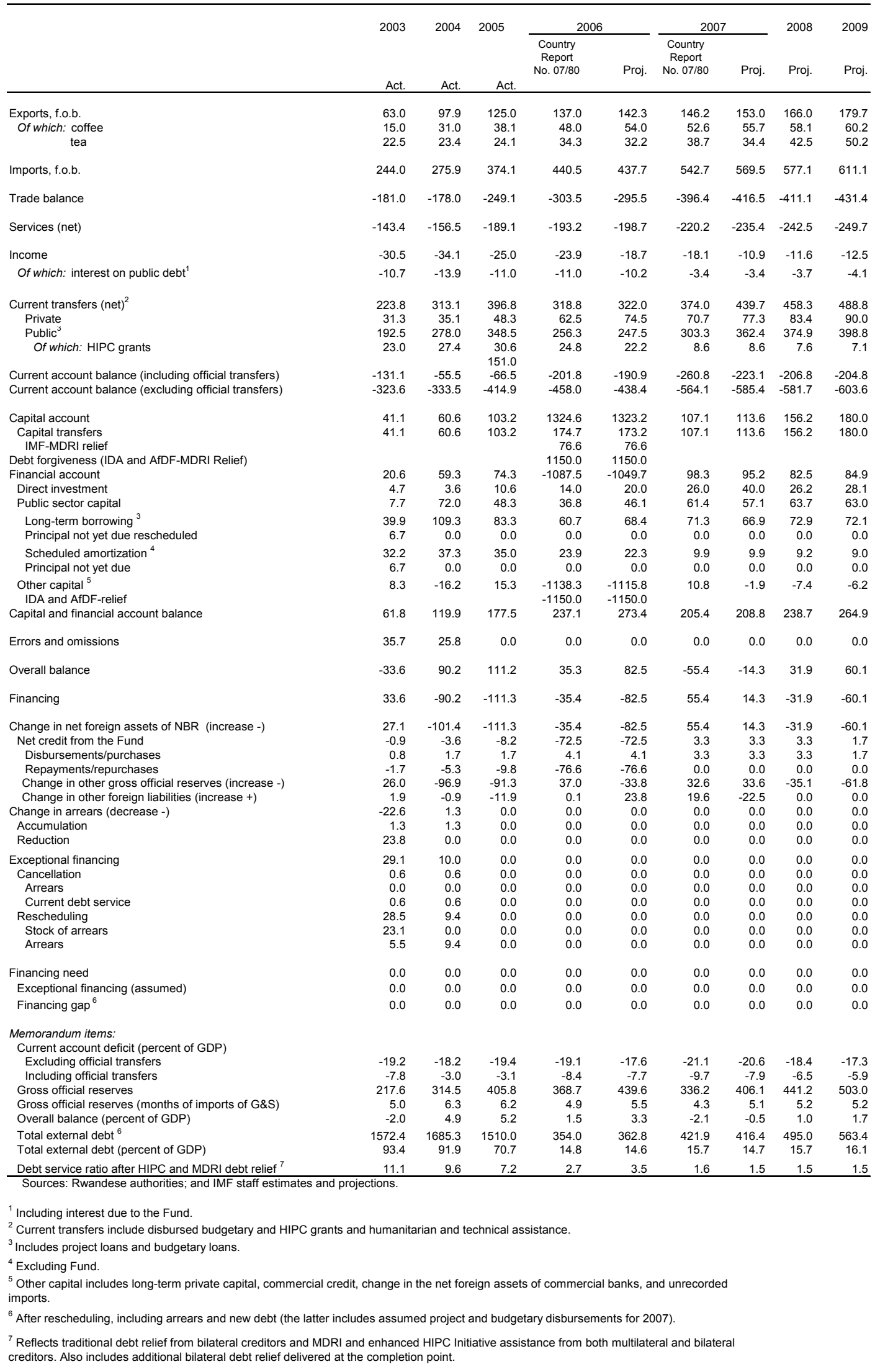


Table 5. Rwanda: Structural Conditionality, 2006-07

\section{Public expenditure management}

Issue draft accounting instructions, forms, and procedures for budget users.

Complete progress report on financial reporting by budgetary agencies (line ministries, provinces, autonomous agencies). Agencies are expected to produce the following monthly financial reports: (i) bank reconciliation statement; (ii) budget execution reports, and (iii) statements of revenue and expenditure.

Publish on the Ministry of Finance's website the progress report on the OBL implementation.

Complete and publish on the Ministry of Finance's website a study on project accounts (for projects listed in paragraph 46 of the TMU) at the NBR (1) showing annual budgets with donor disbursements and spending for 2004-2006 as well as quarterly projections (including a breakdown into imports and the domestic component) for July 2007-June 2008; and

(2) providing an analysis of the causes for the large accumulation of the specific project accounts since January 1 , 2004.

\section{Civil service reform}

Undertake a comprehensive review of the wage structure of the public sector, including fringe benefits and wages included in transfers to local governments.

\section{Private sector development}

Make operational the Office of the Registrar of Land Titles.

\section{Poverty reduction}

Produce a poverty profile of provinces, districts, and communities of Rwanda using the results of the census and the household living conditions survey.

\section{Financial sector}

Submit to Parliament a micro finance law to bring the legal framework for microfinance broadly in line with the Guiding Principles on Regulation and Supervision of Microfinance Institutions as published by the Consultative Group to Assist the Poorest.

\section{Agricultural sector}

Adopt and publish a national fertilizer distribution strategy.
December 31, 2006

June 15, 2007

Benchmark

Not met. Implemented in May 2007.

Benchmark

September 30, Benchmark 2007

September 30, 2007

Performance

Criterion
Not met. Converted to September 30, 2007 benchmark.
October 31, 2007 Benchmark

September 30, Benchmark 2007

June 30, 2007

Benchmark
February 28, Benchmark
Not met. Implemented in April 2007. 
Table 6. Rwanda: Proposed Schedule of Disbursements Under the PRGF Arrangement, 2007-09

\begin{tabular}{lr} 
Date Conditions necessary for disbursement & Amount \\
& $\begin{array}{r}\text { (In millions (In percent } \\
\text { of SDRs) of quota) }\end{array}$ \\
\hline
\end{tabular}

June 25, 2007

Observance of performance criteria for

December 31, 2006, completion of the second

review under the arrangement

October 15, 2007

Observance of performance criteria for June 30,2007 , completion of the third review under the arrangement

April 15, 2008

Observance of performance criteria for

1.14

December 31, 2007, completion of the fourth review under the arrangement

October 15, 2008

Observance of performance criteria for June 30,2008 , completion of the fifth review under the arrangement

April 15, 2009

Observance of performance criteria for

December 31, 2008, completion of the sixth review under the arrangement 
Table 7. Modification of end-June 2007 Performance Criteria on the Domestic Fiscal Balance and Net Credit to Government

(Billions of Rwandese franc)

\section{Domestic Fiscal Balance}

PC set January 10, 2007

$-46.0$

Adjustors

$-8.2$

Shortfall in grants up to maximum of US\$20m to be offset by expenditure cuts

Emergency Food Imports

2.8

Adjusted PC

$-37.8$

Revised domestic deficit

$-56.6$

Discrepancy

18.8

Percent of GDP

Proposed PC

$-64.9$

\section{Net Credit to Government}

PC set January 10, 2007

$-49.8$

Adjustors

Shortfall between actual and baseline grants of US $\$ 20.5 \mathrm{~m}$ to be financed by a drawdown of deposits

Emergency Food Imports

Adjusted PC

Revised NCG

Discrepancy

Percent of GDP

Proposed PC

Source: Rwandese authorities; and staff estimates and projections. 
Mr. Rodrigo de Rato y Figaredo

Managing Director

International Monetary Fund

Washington, D.C. 20431

U.S.A.

Dear Mr. de Rato:

1. The first review of our financial and economic program supported under the Fund's Poverty Reduction and Growth Facility (PRGF) was completed on January 29, 2007. In the attached Memorandum of Economic and Financial Policies (MEFP), we review recent economic developments and amend our policies for the remainder of 2007.

2. Program implementation under the PRGF arrangement has been broadly on track. All quantitative performance criteria for end-December 2006 were met with the exception of the performance criterion (PC) on net credit to the government. As foreshadowed in our MEFP of January 10, 2007, the PC on net credit to government was not met because of delays in reimbursements from the African Union (AU) for our peacekeeping efforts in Darfur. We have agreed with the AU on a schedule of reimbursements and expect no further delays in 2007. Should further delays occur, we stand ready to cut other expenditures to meet the limits on net credit to government. Thus, as the nonobservance was of a temporary nature, we are requesting a waiver for the nonobservance of the quantitative $\mathrm{PC}$ on net credit to the government.

3. In support of our policies described in the MEFP, the Government of Rwanda requests the completion of the second review under the PRGF arrangement and the disbursement of the third loan of SDR 1.14 million.

4. The Government of Rwanda believes that the policies set forth in the attached MEFP are adequate to achieve the objectives of its program, but it will take any further measures that may become appropriate for this purpose. The Government of Rwanda will consult with the Fund on the adoption of these measures and in advance of revisions to the policies contained in the MEFP, in accordance with the Fund's policies on such consultations. The third review under the PRGF arrangement is expected to be completed by no later than end-November 2007, and the fourth review by mid-April 2008. 
5. The Government of Rwanda authorizes the publication and distribution of this letter and MEFP together with the related staff report.

Sincerely yours,

/ s /

François Kanimba

Governor

National Bank of Rwanda
/ s /

James Musoni

Minister of Finance and

Economic Planning

Attachments: Memorandum of Economic and Financial Policies

Technical Memorandum of Understanding 


\section{APPENDIX I-ATTACHMENT I}

\section{Memorandum of ECONOMiC ANd FinanCial Policies (MEFP) OF THE Government OF RWANDA}

June 7, 2007

1. This memorandum amends our medium-term economic program, which was set out in the MEFPs of June 2006 and January 2007 and which is supported by an arrangement under the IMF's Poverty Reduction and Growth Facility. The program reduces poverty by preserving macroeconomic stability, boosting economic growth, and adopting policies that will improve the lives of the poor. This memorandum is consistent with the goals established in the Poverty Reduction and Strategy Paper (PRSP) and three annual progress reports. It is also in line with ongoing preparations for the new Economic

Development and Poverty Reduction Strategy (EDPRS), expected to be finalized by end-year. The memorandum reviews the recent performance under the program and describes the policies for the remainder of 2007 and the medium term.

\section{RECENT ECONOMIC DEVELOPMENTS}

2. With policies remaining on track, macroeconomic performance in 2006 was satisfactory. Despite adverse weather conditions depressing agricultural output, growth at over 5 percent exceeded projections because of buoyant activity in manufacturing and the services sector. However, rising food and energy costs pushed inflation to almost 12 percent at end-2006 (inflation excluding these items remained at about 5 percent).

3. Fiscal policies were in line with the program. Higher-than-programmed revenue offset unforeseen spending, so that the end-2006 target on the domestic fiscal balance was met. The revenue overperformance reflected mostly one-off VAT collections and nontax receipts. On the spending side, there were additional outlays for export promotion, the Kigali Water Project, and construction and repair of schools. As a result, priority spending substantially exceeded the target. However, as foreshadowed in our MEFP of January, the target on net credit to government was missed, because of continued delays in reimbursements from the African Union for our peace keeping efforts in Sudan. ${ }^{1}$

4. Though the end-2006 reserve money target was met, monetary management was complicated by

- an acceleration in fiscal spending, including for projects, toward the end of the year, which necessitated large sterilization operations of the National Bank of Rwanda

\footnotetext{
${ }^{1}$ The amounts owed are about $1 / 2$ percent of GDP.
} 
(NBR). To bring reserve money within the 2006 target, the NBR issued domestic papers, implying a sharp increase in domestic debt in the last quarter.

- $\quad$ the continued rapid expansion in broad money. To a greater extent, this reflected an increase in private sector credit (by 24 percent), mostly for investment. Broad money was also boosted by temporary deposit increases such as a repatriation of an investor, who is expected to reinvest this money gradually in the future.

5. The current account deficit was tighter than programmed. This was due to a substantial increase in private transfers and slightly better export receipts from higher volumes. The NBR's international reserves surpassed the program target, exceeding five months of imports, because project disbursements were higher than programmed and the NBR used an overdraft facility at the Belgian central bank. The nominal effective exchange rate depreciated by 3 percent in 2006, while there was a moderate real appreciation of 4 percent.

6. Early indications for $\mathbf{2 0 0 7}$ are mixed. Inflation declined to 10 percent in April, but inflation excluding food and energy increased to 7 percent, owing to increases in health and education fees and the pass-through of higher food prices to restaurants. The first harvest's production was below the one in 2006 with large shortfalls in some crops, while others overperformed; rains for the mid-year harvest so far have been favorable. Preliminary fiscal data for the first quarter indicate that, despite revenue overperformance covering unforeseen spending, the benchmarks on the domestic deficit and the net credit to government were missed. This is because delays in one EU tranche and the parliamentary approval of the World Bank's Poverty Reduction Support Grant (PRSG) would have triggered an automatic adjuster requiring spending cuts; the PRSG has now been disbursed and the EU grant is expected to come in the second quarter of 2007.

\section{On the structural front:}

- $\quad$ To enhance public financial management, workshops to facilitate the implementation of the Organic Budget Law (OBL) were held. We also decentralized the authority to effect expenditure commitments and virements to budget agencies and Parliament passed the Public Procurement Code and created an intergovernmental fiscal relations unit, whose role, among others, is to report on local government finances. In May, consolidated government financial statements for 2006 were completed, and the Ministry of Finance issued accounting instructions, forms, and procedures for budget users (end-December benchmark). However, the comprehensive review of the wage structure of the public sector (end-December benchmark) has been delayed because we realized that technical assistance is needed (new end-September benchmark). In June, the Auditor General completed an audit of our peace keeping activities (prior action).

- To increase agriculture productivity, we developed and published in April a national fertilizer strategy (end-February benchmark). 
- $\quad$ To develop the foreign exchange market, the NBR revised the foreign exchange regulations and relaxed auction rules.

\section{Medium-Term Policies}

8. The EDPRS will use the findings of recent socio-economic surveys to accelerate poverty reduction. Most importantly, we will intensify efforts to tackle extreme poverty by improving food security and the targeting of job creation and social protection schemes. To this end, we are developing on a pilot base the grassroots "Umurenge" scheme. For small villages, Umurenge will consolidate existing social protection schemes toward income generation for food security and building savings for village investments. Because poverty remains concentrated in rural areas, increasing agricultural productivity will become key and we expect to finalize our agricultural strategy in time for implementation with the 2008 budget. We are also in the process of resolving any discrepancies between the surveys and other data sources, so that the EDPRS will be based on a sound monitoring system, which will assist in the coordination and targeting of policies.

\section{The Program fOR 2007}

9. The objectives of the $\mathbf{2 0 0 7}$ program remain broadly unchanged. We assume a real growth rate of $4 \frac{1}{2}-6 \frac{1}{2}$ percent. With a good mid-year harvest, inflation is expected to decline to 5 percent, while international reserves would cover about 5 months of imports.

Macroeconomic and structural policies are designed to improve the living standards of the poor and promote growth-enhancing investments and reforms.

\section{A. Macroeconomic Program}

\section{Accommodating a substantial fiscal expansion from a scaling up of aid will} require prudent management of macro policies. We had envisaged an increase in the domestic deficit (excluding spending on demobilization and peace keeping) by 1.3 percent of GDP compared with 2006, but the deficit could expand up to 3.3 percent of GDP:

- New grants for education spending, in particular from the Fast Track Education Initiative, are expected to become available. Utilizing these grants will entail an additional increase in the deficit by 1.2 percent of GDP.

- We would increase the deficit further by up to 0.5 percent of GDP for contingent spending. Higher grants of 0.1 percent of GDP have been identified and related spending included in the program. Should more grants materialize, the program would automatically by adjusted by another 0.4 percent of GDP. ${ }^{2}$

\footnotetext{
${ }^{2}$ An increase beyond 3.3 percent of GDP compared with 2006 would be done only in the context of the third review under the PRGF of macroeconomic developments.
} 
- With large shortages in crops, which could have been stored to bridge the time to the next harvest, we will import maize and beans (amounting to about 0.3 percent of GDP) to ensure food security in all districts. This will trigger an automatic adjuster in the program, allowing an increase in the deficit and a commensurate reduction in net foreign assets.

This large fiscal expansion is expected to result in pressures for a real exchange appreciation. To ensure that these pressures do not jeopardize macroeconomic stability, we have put in place a safeguard, which we describe in paragraph 11 .

Fiscal Program, 2006 - 07

(Percent of GDP)

\begin{tabular}{lrrrr}
\hline & $\begin{array}{c}\mathbf{2 0 0 6} \\
\text { Program }\end{array}$ & $\begin{array}{c}\mathbf{2 0 0 6} \\
\text { Actual }\end{array}$ & $\begin{array}{c}\mathbf{2 0 0 7} \\
\text { Program }\end{array}$ & $\begin{array}{c}\mathbf{2 0 0 7} \\
\text { Projected }\end{array}$ \\
\hline Revenue & 15.0 & 15.1 & 15.0 & 14.7 \\
& & & & \\
Expenditure & 21.9 & 21.3 & 22.9 & 23.8 \\
$\quad$ Priority & 10.9 & 11.0 & 12.5 & 13.6 \\
$\quad$ Of which: Emergency food imports & & & & 0.3 \\
$\quad$ Nonpriority & 8.9 & 8.7 & 8.8 & 8.7 \\
Interest & 0.8 & 0.6 & 0.6 & 0.5 \\
Peace keeping support & 0.8 & 0.8 & 0.7 & 0.7 \\
$\quad$ Demobilization & 0.5 & 0.2 & 0.3 & 0.3 \\
& & & & \\
Domestic Fiscal deficit (excluding AU & -5.6 & -5.1 & -6.8 & -8.1 \\
peacekeeping and demobilization) & & & & \\
\hline
\end{tabular}

\section{Fiscal Policy}

11. We have established a safeguard to make the fiscal expansion consistent with the program's inflation objective. Prudent management of scaled-up aid must focus on preventing a crowding out of the private sector, building up costly domestic debt or rekindling inflation. We will thus start monitoring an indicative target on domestic debt. Should the target be exceeded, this would signal that the spending increase was too rapid, forcing the NBR to resort to sterilize the liquidity impact from the additional spending through domestic paper. In this case, the Ministry of Finance would adopt a more gradual approach in releasing the domestic component of the budget in the following quarter.

12. Though the program automatically accommodates grant-financed and foodrelated spending, we request a modification of the end-June targets on the domestic 
fiscal balance and net credit to government. This is because there is a timing issue with several unforeseen spending items taking place in the first half of the year and corresponding financing through grants and higher revenue coming in the second half of the year.

\begin{tabular}{|c|c|c|c|}
\hline \multicolumn{4}{|c|}{$\begin{array}{l}\text { Changes to the } 2007 \text { program } \\
\text { (Billions of Rwandese franc) }\end{array}$} \\
\hline & Jan - Jun & Jul - Dec & Jan - Dec \\
\hline New Resources & -11.9 & 42.9 & 31.0 \\
\hline Higher Revenue & 4.5 & 5.0 & 9.5 \\
\hline Change in Grants & -16.5 & 37.9 & 21.4 \\
\hline$A f D B$ & -18.1 & 18.1 & 0.0 \\
\hline Fast Track Education Initiative & 1.3 & 12.9 & 14.2 \\
\hline Other Education grants & & 3.6 & 3.6 \\
\hline Contingent grants & 1.3 & 1.3 & 2.6 \\
\hline New Spending & 15.1 & 20.2 & 35.3 \\
\hline Priority & 8.5 & 20.7 & 29.1 \\
\hline Fast Track Education Initiative and other & 1.3 & 16.5 & 17.8 \\
\hline Priority Communication & 1.1 & 0.0 & 1.1 \\
\hline Lake Kivu pilot project & 0.0 & 1.0 & 1.0 \\
\hline Food imports & 2.8 & 2.8 & 5.5 \\
\hline Emergency spending for flood victims & 2.4 & 0.4 & 2.8 \\
\hline Emergency rehabilitation of electricty transmission & 0.9 & 0.0 & 0.9 \\
\hline Sterilization cost to NBR & 2.4 & 1.8 & 4.2 \\
\hline Nonpriority & 4.3 & -2.3 & 2.0 \\
\hline Tanzania Refugees & 0.8 & 1.2 & 2.0 \\
\hline Purchase of Banque de Kigali & 3.5 & -3.5 & 0.0 \\
\hline Higher domestic deficit ${ }^{1}$ & -10.6 & -15.3 & -25.9 \\
\hline $\begin{array}{l}\text { Automatic program adjustments for changes in grants and } \\
\text { emergency food imports as of the TMU of January } 10,2007^{2}\end{array}$ & -8.2 & 35.3 & 27.1 \\
\hline Adjusted domestic deficit & -18.8 & 19.9 & 1.1 \\
\hline
\end{tabular}

\section{On the revenue front, we will at least maintain the revenue-to-GDP ratio of}

14.7 percent of GDP (excluding several one-off items of about 0.4 percent of GDP received in the last quarter of 2006, such as from arrears collection and the sale of government cars). As stated in the January MEFP, we stand ready to increase the petroleum excise, should there be any revenue shortfalls.

14. Almost all new spending is for priorities. About half is for new school buildings and laboratories, the purchase of education materials and wages for teachers under the umbrella of the Fast Track Education Initiative. Other priority items include food imports, additional 
funds to complete the Lake Kivu pilot project and emergency assistance to flood victims in Western Province for property and infrastructure repairs. Nonpriority spending increases due to an underestimation of the domestic interest bill and outlays for the resettlement of refugees from Tanzania, but will be financed through higher-than-programmed revenue.

\section{On specific spending items:}

- We are awaiting confirmation from the AU to reimburse us in a timely manner for our peace keeping efforts, particularly because disbursements in January-March were not made on time. Should there be any further delays, we will cut other spending to meet the limits on the net credit to government.

- $\quad$ The government acquired the majority share in the commercial bank Banque de Kigali in February (about 0.3 percent of GDP). With the sale to be completed by year-end, the transaction is not expected to affect our deficit target (see paragraph 24).

- $\quad$ Contingent spending of Rf 4 billion (0.3 percent of GDP) for road maintenance (not included in the projections) is expected to be financed either by additional grants or an increase in the levy for the road fund.

\section{Fiscal structural reforms}

\section{We are in the process of improving our projections for externally-financed} project accounts. Focusing on the largest project accounts as specified in paragraph 46 of the technical memorandum of understanding of January 10, 2007, we will undertake quarterly reconciliations of actuals with projections of project spending, including its import component, starting in May. The first reconciliation report will be issued by June.

\section{The monitoring of local governments is being strengthened. A Prime Minister's} instruction of May 9, 2006 allows the treasury to withhold transfers to provinces and districts ("local governments"), which hold unused balances or which have not been compliant with monthly reporting requirements. Those local governments, which were delinquent in reporting requirements for January and February of 2007 (which were due in March), were informed in mid April that, unless they were compliant by mid-June, their funds would be cut off in July. The first consolidated execution report showing transfers and budget execution per major line items per district and per province during January-April will be published on the Ministry of Finance's website by end-July (performance criterion).

\section{The medium-term Public Financial Management (PFM) action plan is scheduled} for completion by end-2007. ${ }^{3}$ Several measures identified for completion in 2007 in the

\footnotetext{
${ }^{3}$ The PFM has benefited from the Public Expenditure and Financial Accountability (PEFA) assessment conducted in March, and reflects assistance pledged by our development partners.
} 
interim action matrix have been revised with more specific objectives, realistic timelines, and proper sequencing. For each component, we now include ways to measure progress, capacity requirements and institutional responsibilities. In addition to our commitments specified in the January MEFP, which include, in particular, efforts to ensure full implementation of the OBL and financial regulations:

- $\quad$ Public Accounts. We will produce by mid-June 2007, monthly financial reports covering January to March 2007 of all line ministries, provinces, and autonomous agencies: (i) bank reconciliation statement; (ii) budget execution reports, and (iii) statements of revenue and expenditure.

- Budget Preparation. We will complete, by September 2007, a diagnostic review of the Medium-Term Expenditure Framework (MTEF) process, including the integration of the development and recurrent budget. This will assist in determining technical assistance needs for implementation of the MTEF with the 2009 budget framework.

- $\quad$ Budget Execution: To facilitate the move to a treasury single account for local governments, we will develop by June 2007 new procedures to manage decentralization for cash management, commitment control, virements and banking arrangements.

- Auditor General. In collaboration with donors, we continue to strengthen the capacity of the Office of the Auditor General (OAG) through training of staff.

- Internal Audit. We will by December 2007 review the structure of the Internal Audit Department; train internal audit staff on new PFM legal framework; and prepare internal audit manuals with assistance from the World Bank and AFRITAC.

\section{Monetary Policy}

19. Reserve money will remain the operational target to control inflation. Due to an increase in money demand, including through the introduction of new financial instruments, the reserve money trend gradually has deviated from the program over the last years. ${ }^{4}$ In order to improve monetary management, we thus rebased the reserve money program and also made the money anchor more effective by monitoring it now on a monthly instead of quarterly frequency. Based on these adjustments, the NBR will limit year-end reserve money growth to 13 percent. This policy is expected to reduce inflation to 5 percent and to create room for an expansion of credit to the private sector by 17 percent. The NBR will also tighten the management of excess reserves of commercial banks to encourage a stronger interbank

\footnotetext{
${ }^{4}$ At end-2006, the reserve money target was met by lowering banks' reserves substantially below the reserve requirement.
} 
market. To this end, it will mop up excess liquidity, in particular through sales of foreign exchange.

20. To enhance flexibility in the exchange rate, we will develop the interbank foreign exchange market. Our interventions will accommodate upward pressures on the exchange rate through nominal appreciation by raising foreign exchange sales while smoothing out short-term market fluctuations. To enhance flexibility in the exchange rate in the long term, we will discuss with banks the preparations for an exit from the current auction system toward a fully-fledged interbank market.

21. The NBR is committed to maintaining the principles of good governance and best practices. As part of this process, the NBR will publish its financial accounts by June 2007.

\section{External Sector}

22. Because fiscal spending is increased, we expect the external current account deficit (excluding official transfers) to widen to more than 20 percent of GDP (an increase of almost 3 percent of GDP over 2006). This is mainly driven by an expansion in the fiscal deficit, along with the drawdown in projects accounts, and foreign direct investment, which is projected to result in a substantial increase in imports by about 30 percent. Export receipts are expected to increase by about 8 percent, driven mainly by increased volumes in coffee and tea.

\section{We are developing an implementation roadmap for joining the EAC Customs} Union Protocol by June 2009. Initial efforts will be on policy harmonization with a particular focus on developing a list of sensitive products (including those, where higher tariffs could adversely affect the poor). Jointly with other EAC members, we will also seek concessions on industrial imports from non-EAC countries and negotiate the elimination of nontariff barriers.

\section{B. Structural Policies}

\section{Financial sector}

24. The central bank is strengthening the capital base of the banking system.

To improve banks' capital base and tighten the licensing criteria for bank, the minimum capital requirement for banks will be increased on January 1, 2008. Noncompliant banks have provided plans on how to go ahead, including possible merger scenarios. Disinvestment of the Banque de Kigali is well under way and the bidding process for its sale has commenced. To manage the Banque de Kigali during the transition period, the central bank together with the Ministry of Finance signed a memorandum of understanding with the interim management of the bank. The NBR is also discussing with potential new shareholders a capital injection in a problem bank. 
25. The implementation of the Financial Sector Development Plan (FSDP), which we drafted in coordination with FIRST, is being coordinated with donors. The FSDP features

- $\quad$ Strengthening NBR's supervisory powers. While parliament is discussing the amendments to the banking, the central bank has started drafting regulations. Following the parliamentary passage of the Anti-Money Laundering Law, we will establish the appropriate infrastructure for its enforcement, such as setting up a Financial Intelligence Unit.

- $\quad$ Expanding access to credit and financial services. With access to financial services limited outside Kigali, the process of transforming the Union Banques Populaires du Rwanda (UBPR) - a large microfinance network - into a commercial bank is underway. By being able to offer payments services, this would substantially increase access to banking services.

- $\quad$ Long-term finance and capital markets. To facilitate the development of a capital market and provide adequate regulation for market participants, we will, among other: (i) reissue a portion of existing t-bills as bonds and securitize a portion of the longterm debt owed by the government to the Caisse Social du Rwanda to create a yield curve and avenues for longer-term investments for institutional investors such as the pension fund; (ii) develop legal regulatory and operational guidelines; and (iii) establish a Capital Markets Advisory Council to oversee the debt and equity markets.

- Contractual saving institutions. Rwanda's insurance industry and pension fund are an important element of mobilizing long-term funds essential for our development strategy. To this end, we are preparing with the assistance of donors a consolidated regulation and supervision plan for nonbank financial institutions (NBFIs).

- $\quad$ Payments system. To create an effective payments infrastructure, we will, among other, (i) develop a national strategy on payments; and (ii) establish an automated clearing house.

26. We are exploring various options to further boost electricity supply. Electricity generation has now been stabilized thanks to rental generators; at end-2007, a heavy-fuel oil generator is expected to replace some of the expensive rentals. Electrogaz' management is being strengthened and its financial situation is expected to improve in the medium term. A structured tariff has been introduced, and further adjustments will be made when cheaper power sources will become available. We expect the Lake Kivu methane gas pilot project to be operational by end-2007, but the expansion of the project might be delayed due to a legal dispute with other shareholders. In the meantime, we are discussing with various investors new projects related to the lake's gas reserves. Feasibility studies and discussions on financing options for various hydro dams are ongoing, including on a regional basis. 
There are also more than 30 microdam projects in progress. In parallel, we are working on designing the transmission and distribution networks to increase access.

\section{The pace of investment climate reforms in the justice and land sectors is being} accelerated. A three-year project started in May 2007 in partnership with the private sector and with funding from the Investment Climate Facility for Africa (ICF). To address legal and regulatory constraints facing businesses, the project focuses on commercial dispute resolution, and business and land registration reform.

28. The Statistical Institute is revising national accounts. Reflecting a more recent base year (2001), we have improved the coverage of survey data, particularly on the services sector. As a result, greater consistency has been achieved between national accounts and poverty statistics. As a next step, we will be reviewing the methodology for calculating the GDP deflator.

\section{Program Monitoring}

29. Conditionality and program reviews. The third review of the PRGF arrangement, scheduled for completion by November 31,2007, will review quantitative performance as of June 2007 and structural conditionality through September 2007. It will focus on expenditure management and financial sector reform. We have set new structural conditionality on the monitoring of project accounts and the delayed comprehensive review of the wage structure of the public sector.

30. Technical memorandum of understanding (TMU). The attached TMU lays out the details of program design and terminology. We have kept the broad design of the program for 2006 but have (1) modified the definition and the quantitative end-June and end-December 2007 targets of the performance criterion on reserve money as well as the indicative targets on broad and extended broad money; (2) added an indicative target on domestic debt to ensure that the private sector is not crowded out by a fiscal expansion; and (3) modified the end-June quantitative performance criteria on the domestic fiscal deficit and net credit to government and introduced a new adjuster to the domestic fiscal deficit. 
Table 1. Rwanda: Quantitative Performance Criteria and Benchmarks for 2006

(RF billions, unless otherwise indicated)

(Quantitative benchmarks* and performance criteria on test dates ${ }^{* *}$ )

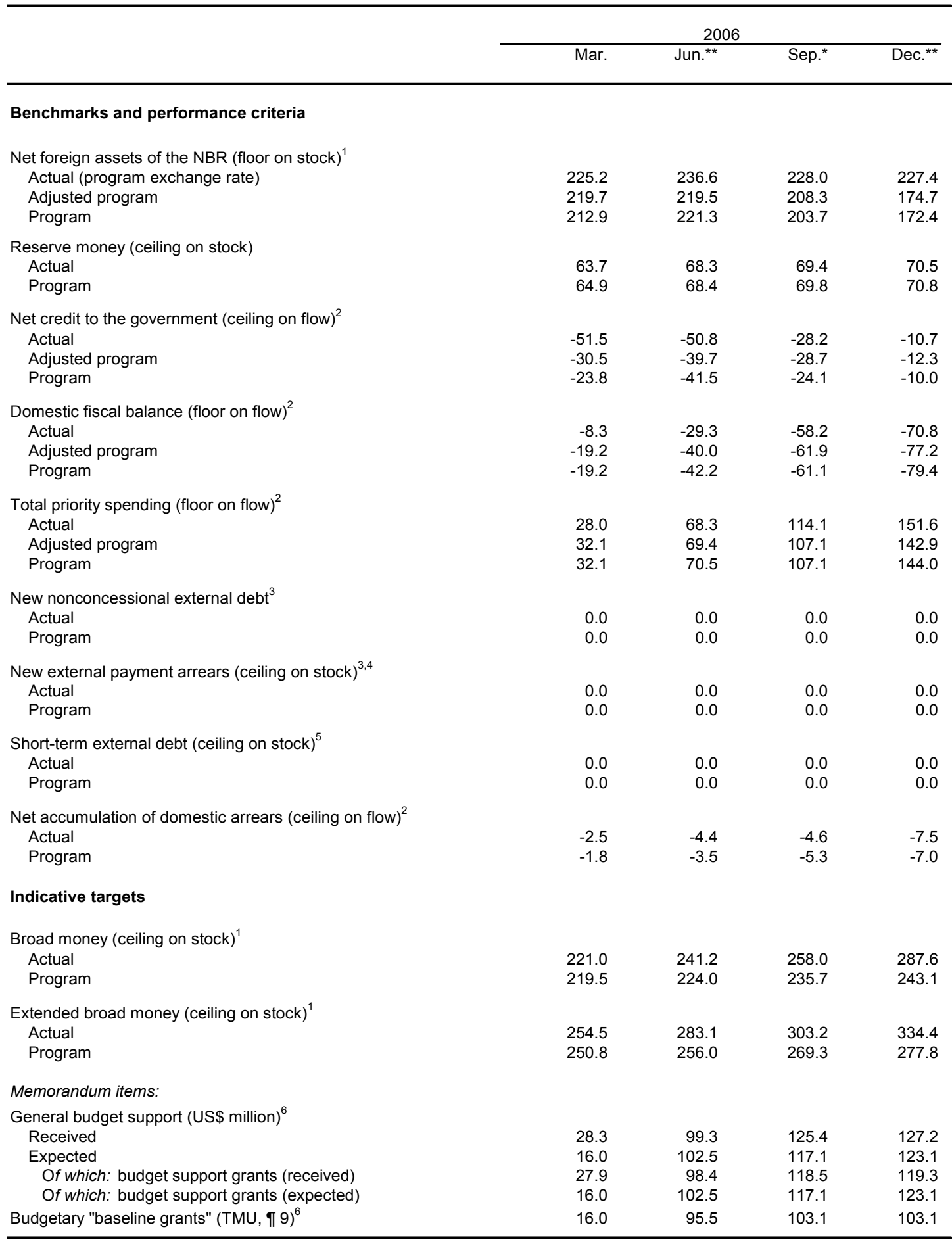

Sources: Rwandese authorities and IMF staff estimates and projections.

${ }^{1}$ At the program exchange rate of RF 553.7/US\$.

${ }^{2}$ Numbers are cumulative from December 31, 2005.

${ }^{3}$ This is a continuous performance criterion.

${ }^{4}$ Excludes arrears on obligations that are subject to rescheduling.

${ }^{5}$ Ceiling on outstanding stock of external debt (excluding normal imported-related credits) owed or guaranteed by the central government, local government, or the NBR with original maturity of up to, and including one year.

${ }^{6}$ Excluding external donor financing for demobilization and peacekeeping.

\section{CInternational Monetary Fund. Not for Redistribution}


Table 2. Rwanda: Quantitative Performance Criteria and Benchmarks for $2007^{1}$ (Billions of Rwanda francs, unless otherwise indicated)

(Quantitative benchmarks ${ }^{\star}$; and performance criteria on test dates ${ }^{\star *}$ )

\begin{tabular}{|c|c|c|c|}
\hline \multirow{3}{*}{ Benchmarks and performance criteria } & \multicolumn{3}{|c|}{2007} \\
\hline & \multirow[t]{2}{*}{ Jun. $^{* *}$} & Sep. ${ }^{*}$ & Dec. $^{\star \star}$ \\
\hline & & & \\
\hline Net foreign assets of the NBR (floor on stock) ${ }^{2}$ & & & \\
\hline Actual (program exchange rate) & & & \\
\hline Adjusted program & & & \\
\hline Program & 244.3 & 206.8 & 179.3 \\
\hline \multicolumn{4}{|l|}{ Reserve money (ceiling on stock) ${ }^{3}$} \\
\hline Actual & & & \\
\hline Program & 83 & 88.1 & 87.9 \\
\hline \multicolumn{4}{|l|}{ Net credit to the government (ceiling on flow) ${ }^{4}$} \\
\hline \multicolumn{4}{|l|}{ Actual } \\
\hline \multicolumn{4}{|l|}{ Adjusted program } \\
\hline Program & -37.7 & -25.7 & 4.7 \\
\hline \multicolumn{4}{|l|}{ Domestic fiscal balance (floor on flow) ${ }^{4,5}$} \\
\hline \multirow{2}{*}{\multicolumn{4}{|c|}{$\begin{array}{l}\text { Actual } \\
\text { Adjusted program }\end{array}$}} \\
\hline Adjusted program & & & \\
\hline Program & -64.9 & -70.6 & -98.7 \\
\hline \multicolumn{4}{|l|}{ Total priority spending (floor on flow) ) $^{4,5}$} \\
\hline \multirow{2}{*}{\multicolumn{4}{|c|}{ Actual }} \\
\hline \multirow{2}{*}{\multicolumn{3}{|c|}{$\begin{array}{l}\text { Adjusted program } \\
\text { Program }\end{array}$}} & \\
\hline & & 132.4 & \\
\hline \multicolumn{4}{|l|}{ New nonconcessional external debt ${ }^{6}$} \\
\hline \multicolumn{4}{|l|}{ Actual } \\
\hline Program & 0.0 & 0.0 & 0.0 \\
\hline \multicolumn{4}{|l|}{ New external payment arrears (ceiling on stock) ${ }^{6,7}$} \\
\hline \multicolumn{4}{|l|}{ Actual } \\
\hline Program & 0.0 & 0.0 & 0.0 \\
\hline Short-term external debt (ceiling on stock) ${ }^{8}$ & & & \\
\hline Actual & & & \\
\hline Program & 0.0 & 0.0 & 0.0 \\
\hline Net accumulation of domestic arrears (ceiling on flow) ${ }^{4}$ & & & \\
\hline Actual & & & \\
\hline Program & -3.9 & -5.4 & -7.0 \\
\hline Indicative targets & & & \\
\hline Broad money (ceiling on stock) ${ }^{2}$ & & & \\
\hline Actual & & & \\
\hline Program & 297.7 & 305.3 & 312.4 \\
\hline Extended broad money (ceiling on stock) ${ }^{2}$ & & & \\
\hline Actual & & & \\
\hline Program & 346.2 & 355.0 & 363.3 \\
\hline Net Present Value of the Stock of Outstanding Debt (Millions of US\$ & & & \\
\hline As projected for end-December 2007 & 200.409 & & 200.409 \\
\hline As projected for end-December 2008 & 239.819 & & 239.819 \\
\hline As projected for end-December 2009 & 279.465 & & 279.465 \\
\hline Ceiling on stock of domestic debt ${ }^{10}$ & & & \\
\hline Actual & & & \\
\hline Adjusted program & & & \\
\hline Program & 175.2 & 187.1 & 185.1 \\
\hline Memorandum items: & & & \\
\hline General budget support (Millions of US $\$)^{5,11}$ & & & \\
\hline Received & & & \\
\hline Expected & 186.5 & 193.0 & 196.6 \\
\hline Of which: budget support grants (received) & & & \\
\hline Of which: budget support grants (expected) & 186.5 & 193.0 & 196.6 \\
\hline Budgetary "baseline grants" (TMU, para. 9) ${ }^{11}$ & 176.5 & 178.0 & 176.6 \\
\hline Revised domestic fiscal balance (excluding AU, demob and food) ${ }^{12}$ & -43.3 & -65.1 & -93.2 \\
\hline
\end{tabular}

Sources: Rwandese authorities and IMF staff estimates and projections.

${ }^{1}$ New targets or indicative targets, which were modified compared with Table 2 of the January 10 MEFP, are shaded.

${ }^{2}$ At the program exchange rate of RF 549.9/US\$.

${ }^{3}$ Targets are calculated as an arithmetic average of the stock of reserve money on the last day of each calendar month in the quarter. Program projections are done quarterly. To derive the monthly figures, seasonality factors and a trend are applied to the quarterly projections. The seasonality factors are derived by applying the X11 filter to currency in circulation over 2002-06 (the factors were robust with respect to different time periods). The seasonality factors were 0.93 for April, 0.96 for May, 1.01 for June, 1.0 for July, 0.98 for August, 0.96 for September, 0.94 for October, 0.94 for November and 1.0 for December

${ }^{4}$ Numbers are cumulative from December $31,2006$.

${ }^{5}$ The domestic fiscal balance targets will be adjusted by the amount of grants exceeding or below programmed grants with the adjusters as specified in the TMU of January 10 2007, and paragraph 3 of the TMU of June 7, 2007. Also the priority spending targets will be adjusted by the amount of grants exceeding or below programmed grants.

${ }^{6}$ This is a continuous performance criterion.

${ }^{7}$ Excludes arrears on obligations that are subject to rescheduling.

${ }^{8}$ Ceiling on the stock of external debt (excluding normal imported-related credits) owed or guaranteed by the central governments, local government, or the NBR with original maturity of up to, and including one year.

${ }^{9}$ Figures indicate the NPV projections based on debt contracted at the test date.

${ }^{10}$ Numbers show end of period stocks, at the end of 2006 the stock stood at RF 189.6 billion.

${ }^{11}$ Excluding external donor financing for demobilization and peacekeeping.

12 Used for the calculation of the adjuster on the indicative limits on domestic debt.

\section{CInternational Monetary Fund. Not for Redistribution}


Table 3. New Structural Conditionality, 2007

\section{Prior action for the completion of the second review under the PRGF}

Publish on the Ministry of Finance's public website the Auditor General's audit of peace keeping activities during

Prior action, 2006.

implemented on

June 6, 2007

\section{Public expenditure management}

Undertake a comprehensive review of the wage structure of the public sector, including fringe benefits and wages included in transfers to local governments.

Publish on the Ministry of Finance's website the first consolidated execution report of local governments showing transfers and budget execution per major line items per district and per province for the period January-April.
September 30, 2007

Benchmark

July 31, 2007
Performance

Criterion 


\section{APPENDIX I-ATTACHMENT II \\ RWANDA: TECHNICAL MEMORANDUM OF UNDERSTANDING}

June 7, 2007

1. This technical memorandum of understanding (TMU) supplements the TMU of January 10, 2007. It (1) outlines the changes to the quantitative program targets compared with Table 2 of the MEFP of January 10, 2007; (2) introduces a new adjuster to the domestic fiscal balance; (3) introduces a new quantitative target on domestic debt; and (4) modifies the definition for the quantitative targets on reserve money.

\section{Quantitative Program Targets}

2. The following revisions have been made compared with Table 2 of the MEFP dated January 10, 2007:

- $\quad$ Modified performance criteria for June 30, 2007 and December 31, 2007 as well as indicative targets for September 30, 2007 are proposed to be established with respect to ceilings on reserve money.

- A modified performance criterion for June 30, 2007 is proposed to be established with respect to the floor on the domestic fiscal balance of the central government.

- A modified performance criterion for June 30, 2007 is proposed to be established with respect to the ceiling on the net credit to the central government.

- Indicative targets are proposed to be established for June 30, 2007, September 30, 2007, and December 31, 2007 for ceilings on the consolidated domestic debt of the public sector.

- $\quad$ Modified indicative targets are proposed to be established for June 30, 2007, September 30, 2007, and December 31, 2007 for ceilings on broad money and extended broad money.

All other targets are unchanged from those set in Table 2 of the MEFP of January 10, 2007.

\section{A. Targets Related to the Execution of the Fiscal Program}

\section{Domestic Fiscal Balance of the Central Government (DFB)}

\section{New Adjuster.}

- $\quad$ The floor on the DFB will be adjusted downward (i.e., the deficit target will be increased) by the amount of the positive difference between actual and programmed 
budgetary grants up to a maximum of Rwandese Franc 26.4 billion. The increase in the deficit would be used for spending as specified in bullet 1 of paragraph 9 of the TMU of January 10, 2007. If actual grants exceed programmed grants by more than Rwandese Franc 26.4 billion, the floor on the DFB will be adjusted downward in the context of the third review of the PRGF with Fund staff IF this review establishes that a further fiscal expansion would not lead to a rekindling of inflation or an undue increase in domestic debt.

\section{Domestic debt of the central government and the National Bank of Rwanda (DD)}

4. A ceiling applies to the DD for the end-June 2007, end-September 2007, and endDecember 2007 targets.

5. Definition. Domestic debt of the central government and the National Bank of Rwanda includes the stock of treasury bills for government financing at coupon value, plus government borrowing from public enterprises (FinaBank formerly Bank Continental Africaine du Rwanda, Bank Commerciale du Rwanda, Bons de Developpement, Credit Savings Scheme, Caisse Social du Rwanda, OCIR-Thé; and rescheduling of old arrears) plus the stock of treasury bills issued for monetary policy, plus any one-day or one-week deposits of commercial banks at the central bank excluding excess and required reserves. ${ }^{1}$ The stock of domestic debt was Rf 189.6 billion at end-2006.

\section{Adjusters.}

In the case of a grant-financed expansion of the domestic fiscal balance to limit the crowding out of the private sector through sterilization: The ceiling on domestic debt will be adjusted (both upward and downward) by one-third of the difference between the actual domestic fiscal balance and the revised domestic fiscal balance (excluding AU peace keeping, demobilization and food imports). That is, if the actual deficit is higher than the revised programmed deficit, the ceiling would be revised upward.

- In the case of a shortfall in grants, for which an automatic adjuster would increase the limit on net credit to government: The ceiling on domestic debt will be adjusted upward by any negative difference between actual and "baseline grants" up to a maximum adjustor of US\$30 million, evaluated in Rwanda francs at the program exchange rate (see also paragraph 13 of the TMU of January 10, 2007).

7. Reporting requirement. Data on domestic debt will be transmitted on a monthly basis within four weeks of the end of each month.

\footnotetext{
${ }^{1}$ T-bill holdings of the NBR are excluded because this is the consolidated debt of the central government and the NBR.
} 


\section{B. Targets for Monetary Aggregates}

\section{Reserve Money}

8. Modification of the measurement of the ceiling on the stock of reserve money.

The stock of reserve money for the quarter will be calculated as the arithmetic average (mean) of the stock of reserve money at the last day of each calendar month comprising the quarter. 
Appendix II—Rwanda: Millennium Development Goals

\begin{tabular}{|c|c|c|c|c|c|c|c|c|}
\hline & 1990 & 1995 & 2000 & 2002 & 2003 & 2004 & 2005 & $\begin{array}{l}\text { Likelihood of reaching } \\
\text { the target by } 2015^{\star *}\end{array}$ \\
\hline \multicolumn{9}{|l|}{ Goal 1: Eradicate extreme poverty and hunger } \\
\hline Percentage share of income or consumption held by poorest $20 \%$ & .. & .. & .. &.. &.. & .. &.. & \\
\hline Population below $\$ 1$ a day $(\%)$ &.. & .. & 51.7 & .. & .. & 60 & & Challenging \\
\hline Population below minimum level of dietary energy consumption (\%) &.. &.. & .. & 37 & 37 & .. &.. & \\
\hline Poverty gap ratio at $\$ 1$ a day (incidence $x$ depth of poverty) &.. & .. & 20 & .. & .. &.. & .. & \\
\hline Poverty headcount, national (\% of population) &.. & .. & 60.3 &.. & .. & .. & 56.9 & \\
\hline Prevalence of underweight in children (under five years of age) & .. & .. & 24.3 & .. & .. & .. & .. & Achievable \\
\hline \multicolumn{9}{|l|}{ Goal 2: Achieve universal primary education } \\
\hline Net primary enrollment ratio (\% of relevant age group) & 67.4 & .. & 84 & 86.7 & 86.7 & .. &.. & Likely \\
\hline Primary completion rate, total (\% of relevant age group) & 44 & .. & 22.4 & 37 & 37.3 & 37.4 & & Achievable \\
\hline Proportion of pupils starting grade 1 who reach grade 5 & 59.9 &.. & 40 & .. &.. &.. &.. & \\
\hline Youth literacy rate (\% ages $15-24)$ & 72.7 & 78.6 & 83.4 & 84.9 & & &.. & \\
\hline Goal 3: Promote gender equality and empower women & & & & & & & & Likely \\
\hline Proportion of seats held by women in national parliament (\%) & 17 & .. & 17 & 26 & 26 & 49 & 49 & \\
\hline Ratio of girls to boys in primary and secondary education (\%) & 96.4 & .. & 96 & 94.8 & 98.7 & 100.1 & 100.1 & Achievable \\
\hline Ratio of young literate females to males (\% ages 15-24) & 86.4 & 91.8 & 95.8 & 96.9 & & .. & 97.9 & Likely \\
\hline Share of women employed in the nonagricultural sector (\%) & 16.7 &.. & .. & .. &.. & .. & .. & \\
\hline \multicolumn{9}{|l|}{ Goal 4: Reduce child mortality } \\
\hline Immunization, measles ( $\%$ of children ages $12-23$ months) & 83 & 84 & 74 & 69 & 90 & 84 & 84 & Already Achieved \\
\hline Infant mortality rate (per 1,000 live births) & 103 & 124 & 118 &.. & 118 & 118 & 118 & Achievable \\
\hline Under 5 mortality rate (per 1,000$)$ & 173 & 209 & 203 & .. & 203 & 203 & 203 & Achievable \\
\hline \multicolumn{9}{|l|}{ Goal 5: Improve maternal health } \\
\hline Births attended by skilled health staff (\% of total) & .. & .. & 31.3 & .. & .. & .. & .. & Achievable \\
\hline Maternal mortality ratio (modeled estimate, per 100,000 live births) & .. & .. & 1400 &.. & .. & .. &.. & Unlikely \\
\hline Goal 6: Combat HIVIAIDS, malaria, and other diseases & & & & & & &.. & \\
\hline Contraceptive prevalence rate (\% of women ages 15-49) & .. & .. & 13.2 & .. & .. & .. &.. & Challenging \\
\hline Incidence of tuberculosis (per 100,000 people) & 129.7 & 234.6 & 324.1 & 357.3 & 373.8 & 373.8 & 370.9 & \\
\hline Number of children orphaned by HIVIAIDS & .. & .. & 160000 &.. & 160000 & 160000 & 210000 & \\
\hline Prevalence of HIV, total (\% of population aged 15-49) & .. & .. & &.. & 5.1 & & 3 & \\
\hline Tuberculosis cases detected under DOTS (\%) & .. & 35.5 & 34.2 & 31.1 & 27.5 & 27.5 & 29.4 & Already Achieved \\
\hline \multicolumn{9}{|l|}{ Goal 7: Ensure environmental sustainability } \\
\hline Access to an improved water source ( $\%$ of population) & 58 &.. & .. & 73 & 73 & .. & 74 & Achievable \\
\hline Access to improved sanitation (\% of population) & 37 &.. & .. & 41 & &.. & 42 & \\
\hline Access to secure tenure ( $\%$ of population) &.$\cdot$ &.$\cdot$ &.$\cdot$ & .. & .. &.$\cdot$ & .. & \\
\hline CO2 emissions (metric tons per capita) & 0.1 & 0.1 & 0.1 & .. & .. & .. & .. & \\
\hline Forest area (\% of total land area) & 18.5 &.. & 12.4 & .. & .. &.. &.. & Unlikely \\
\hline GDP per unit of energy use (2000 PPP \$ per kg oil equivalent) & .. & .. & .. & .. & .. & .. & .. & \\
\hline Nationally protected areas (\% of total land area) & .. & .. & .. &.. & 6.2 & 6.2 & 6.2 & \\
\hline \multicolumn{9}{|l|}{ Goal 8: Develop a global partnership for development } \\
\hline Aid per capita (current US\$) & 41.9 & 123.3 & 41.8 & 43.5 & 39.5 & 39.5 & 52.6 & NA \\
\hline Debt service (\% of exports) &.. & .. & .. & .. & 32 & 29.8 & 10 & \\
\hline Fixed line and mobile phone subscribers (per 1,000 people) & 1.7 & 1.3 & 7.3 & 16.4 & 16.4 & .. & 18.2 & \\
\hline Internet users (per 1,000 people) &.. &.. & 0.6 & 3.1 & 3.5 & 4.3 & 4.3 & \\
\hline Personal computers (per 1,000 people) & .. & .. & .. &.$\cdot$ &.$\cdot$ & .. &.$\cdot$ & \\
\hline Unemployment, youth female (\% of female labor force ages $15-24$ ) & .. &.. &.. &.. &.. &.. &.. & \\
\hline Unemployment, youth male (\% of male labor force ages 15-24) &.. &.. & .. &.. & .. &.. &.. & \\
\hline Unemployment, youth total (\% of total labor force ages $15-24$ ) &.. & .. &.. & .. & .. & .. & .. & \\
\hline \multicolumn{9}{|l|}{ Other } \\
\hline Fertility rate, total (births per woman) & 7.1 & .. & 5.9 & .. & 5.6 & 5.5 & 5.5 & \\
\hline GNI per capita, Atlas method (current US\$) & 370 & 220 & 250 & 230 & 200 & 210 & 230 & \\
\hline GNI, Atlas method (current US\$) (billions) & 2.5 & 1.3 & 2.0 & 1.9 & 1.8 & 1.9 & 2.1 & \\
\hline Gross capital formation ( $\%$ of GDP) & 14.6 & 13.4 & 17.5 & 19.0 & 18.4 & 20.8 & 21 & \\
\hline Life expectancy at birth, total (years) & 40.2 &.. & 40.9 & 39.8 & 43.7 & 43.9 & 43.9 & \\
\hline Literacy rate, adult total ( $\%$ of people ages 15 and above) & 53.3 & 60.2 & 66.8 & 69.2 & & 69.4 & 64.9 & \\
\hline Population, total (millions) & 6.9 & 5.7 & 8.0 & 8.2 & 8.8 & 8.9 & 9 & \\
\hline Trade ( $\%$ of GDP) & 19.7 & 31.0 & 32.6 & 33.1 & 36.2 & 36.2 & 41.5 & \\
\hline
\end{tabular}

Source: World Development Indicators Database, April 2006.

Data in italics are estimates.

** World Bank Estimates 
INTERNATIONAL MONETARY FUND

RWANDA

Second Review Under the Three-Year Arrangement Under the Poverty Reduction and Growth Facility, Request for Waiver of Nonobservance of Performance Criterion, and Modification of Performance Criteria-Informational Annex

Prepared by the African Department

(In collaboration with other departments)

Approved by Sharmini Coorey and Mark Plant

June 7, 2007

- Relations with the Fund. Describes financial and technical assistance by the IMF and provides information on the safeguards assessment and exchange system. Outstanding Fund credit was SDR3.99 million (4.99 percent of quota) at end-April 2007. Completion of the second review would allow the disbursement of SDR1.14 million.

- $\quad$ Relations with the World Bank Group. Describes World Bank Group program and portfolio and provides statement of IFC investments.

- $\quad$ Statistical Issues. Assesses the quality of statistical data. Although economic data are generally adequate for surveillance, weaknesses hamper economic analysis. 


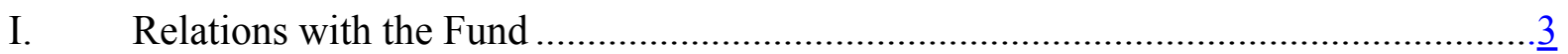

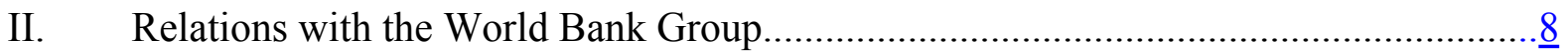

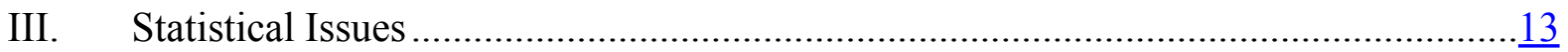

Tables:

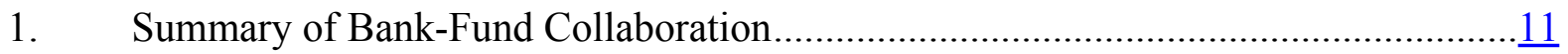

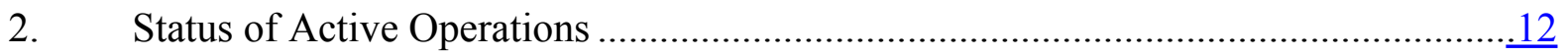

3. Table of Common Indicators Require for Surveillance ...........................................16 


\section{APPENDIX I. RWANDA: RELATIONS WITH THE FUND}

(As of April 30, 2007)

I. Membership Status: Joined: September 30, 1963

Article VIII

II. General Resources Account:

Quota

SDR million
80.10
80.11
0.00

percent Quota

100.00

Fund holdings of currency

100.02

Reserve position in Fund

III. SDR Department:

$\underline{\text { SDR million }}$

percent Allocation

Net cumulative allocation

13.70

100.00

Holdings

15.20

110.99

IV. Outstanding Purchases and Loans:

PRGF arrangements

$\underline{\text { SDR million }}$

3.99

percent Quota

4.99

V. Latest Financial Arrangements:

$\begin{array}{lcccc}\text { Type } & \begin{array}{c}\text { Approval } \\ \text { date }\end{array} & \begin{array}{c}\text { Expiration } \\ \text { date }\end{array} & \begin{array}{c}\text { Amount } \\ \text { approved } \\ \text { (SDR million) }\end{array} & \begin{array}{c}\text { Amount drawn } \\ \text { (SDR million) }\end{array} \\ \text { PRGF } & \text { Jun 12, 2006 } & \text { Jun 11, 2009 } & 8.01 & 2.28 \\ \text { PRGF } & \text { Aug 12, 2002 } & \text { Jun 11, 2006 } & 4.00 & 4.00 \\ \text { PRGF } & \text { Jun 24, 1998 } & \text { Apr 30, 2002 } & 71.40 & 61.88\end{array}$

VI. Projected Payments to the Fund (SDR million; based on existing use of resources and present holdings of SDRs):

Principal

Charges/Interest

Total

$\begin{array}{lccrr}\underline{2007} & \underline{2008} & \underline{2009} & \underline{2010} & \underline{2011} \\ 0.02 & 0.02 & 0.02 & 0.02 & 0.40 \\ 0.02 & 0.02 & 0.02 & 0.08 & 0.42\end{array}$

VII. Implementation of HIPC Initiative:

Enhanced

framework

I Commitment of HIPC assistance

Decision point date

Dec 2000 
Assistance committed by all creditors

(US\$ million) ${ }^{19}$

695.50

Of which: IMF assistance (US\$ million)

63.40

(SDR equivalent in millions)

46.79

Completion point date

Apr 2005

II Delivery of IMF assistance (SDR million)

Amount disbursed

Interim assistance

Completion point

32.34

Additional disbursement of interest income ${ }^{20}$

3.77

Total disbursements

50.56

\section{Implementation of MDRI Assistance}

I Total Debt Relief (SDR Million) ${ }^{21}$

Of which: MDRI

HIPIC

32.55

II Debt Relief by Facility (SDR million)

Delivery Date

January 2006
GRA

NA
PRGF Total

$52.74 \quad 52.74$

Decision point - point at which the IMF and the World Bank determine whether a country qualifies for assistance under the HIPC Initiative and decide on the amount of assistance to be committed.

\footnotetext{
${ }^{19}$ Assistance committed under the original framework is expressed in net present value (NPV) terms at the completion point, and assistance committed under the enhanced framework is expressed in NPV terms at the decision point. Hence these two amounts can not be added.

${ }^{20}$ Under the enhanced framework, an additional disbursement is made at the completion point corresponding to interest income earned on the amount committed at the decision point but not disbursed during the interim period.

${ }^{21}$ The Multilateral Debt Relief Initiative (MDRI) provides 100 percent debt relief to eligible member countries that are qualified for the assistance. The debt relief covers the full stock of debt owed to the Fund as of end2004 which remains outstanding at the time the member qualifies for such debt relief. The MDRI is financed by bilateral contributions and the Fund's own resources, as well as the resources already disbursed to the member under the HIPC Initiative (see Section VII above).
} 
Interim assistance - amount disbursed to a country during the period between decision and completion points, up to 20 percent annually and 60 percent in total of the assistance committed at the decision point (or 25 percent and 75 percent, respectively, in exceptional circumstances).

Completion point - point at which a country receives the remaining balance of its assistance committed at the decision point, together with an additional disbursement of interest income as defined in footnote 3 above. The timing of the completion point is linked to the implementation of pre-agreed key structural reforms (i.e., floating completion point).

\section{Safeguards Assessments:}

Under the Fund's safeguards assessment policy, the National Bank of Rwanda (NBR) was subject to a safeguards assessment with respect of the PRGF arrangement approved on June 12, 2006. The update assessment proposed recommendations to address continuing vulnerabilities in the external audit and financial reporting areas. The implementation of these measures is being monitored by IMF staff.

\section{Exchange System:}

The Rwanda franc was pegged to the SDR until March 6, 1995, when Rwanda adopted a market-determined exchange rate system. However, in the IMF's most recent Quarterly Report on Exchange Arrangements, the exchange rate regime was reclassified to a conventional fixed peg. On December 1998, Rwanda accepted the obligations under Article VIII, Sections 2, 3 and 4 of the IMF, and maintains a system free of restrictions on the making of payments and transfers for current international transactions. In 2001, a foreign exchange auction system was put in place with technical assistance from MFD. Since February 7,2001, auctions have been taking place on a weekly basis and the foreign exchange auctions impose a limit of $+/$ - RF 5 to the margin by which the exchange rate can vary from the previous day.

\section{Article IV Consultation:}

Rwanda is on the revised 24-month consultation cycle. The Executive Board discussed the staff report for the 2006 Article IV consultation (IMF Country Report No. 07/80) on January 29, 2007.

\section{FSAP Participation, ROSCs, and OFC Assessments:}

A Report on Observance of Standards and Codes on Fiscal Transparency (ROSC) was issued in July 2003. A Financial Sector Assessment Program (FSAP) has taken place in February 2005. Rwanda has not had an Offshore Financial Center (OFC) assessment. 


\section{Technical Assistance:}

1999 FAD long-term experts, on tax policy, on budget preparation, and on treasury management.

1999 MFD long-term general advisor to governor of NBR.

1999 MFD experts on banking supervision and foreign exchange market operations.

2000 FAD experts on budget execution and on tax policy.

2000 MFD experts on foreign exchange market operations, and banking supervision.

2000 STA mission on money and banking statistics.

2000 STA mission on balance of payments statistics.

2001 FAD experts on expenditure management and on tax policy.

2001 FAD mission on tax policy.

2001 MFD mission on foreign exchange policy, monetary policy, and banking supervision.

2001 MFD expert on monetary policy implementation.

2001 FAD mission on assessment of tracking of poverty reducing expenditure, and the fiscal ROSC.

2001 MFD expert on banking supervision.

2001 FAD experts on expenditure management, and on tax policy (until mid-year).

2002 MFD expert on banking supervision (until November).

2002 MFD expert on monetary and foreign exchange rate policy.

2002 AFRITAC East work plan mission.

2003 AFRITAC East mission on statistical issues.

2003 AFRITAC East expert on Organic Budget Law.

2003 FAD mission on fiscal ROSC and budget management system.

2003 AFRITAC East mission on developing the market for government treasury bills.

2003 FAD mission on reform of investment incentives and tax reform.

2003 MFD expert on monetary and foreign exchange rate policy.

2003 MFD expert on banking supervision and regulation.

2003 MFD missions on banking supervision.

2003 MFD mission on foreign reserves management.

2003 STA multi-sector statistics mission.

2003 FAD mission on Decentralization.

2003 FAD mission on revenue administration.

2004 AFRITAC East expert on financial regulations.

2004 AFRITAC East mission on treasury reforms.

2004 AFRITAC East expert on implementing cash flow planning and banking arrangements.

2004 FAD tax administration expert on strengthening of revenue administration.

2004 FAD mission on revenue administration.

2004 MFD/LEG mission on Anti-Money Laundering and Combating the Financing of Terrorism. 
2004 MFD expert on banking supervision and regulation.

2004 MFD expert on monetary policy, monetary operations, and money markets.

2004 MFD missions on on-site banking supervision.

2005 AFRITAC East expert follow up on cash management.

2005 MFD/LEG mission on Anti-Money Laundering and Combating the Financing of Terrorism.

2005 MFD mission on financial statements of specific bank.

2005 MFD-WB joint FSAP mission.

2005 LEG mission on customs legislation.

2005 STA mission on Balance of Payments statistics.

2005 LEG mission on tax legislation.

2005 FAD mission on public accounting: decentralized accounting for central government.

2005 FAD expert on tax administration.

2005 MFD resident expert on monetary operations, monetary policy, money markets.

2005 MFD expert on banking supervision.

2005 LEG mission on drafting an amendment to the banking law.

2005 MFD mission on banking supervision and bank restructuring.

2006 AFRITAC East experts on establishing intergovernmental fiscal unit in the MOF.

2006 FAD advisor trade facilitation.

2006 MFD FSAP-follow up advisory mission on monetary and foreign exchange operations and NBR internal audit.

2006 LEG mission to strengthen legal and regulatory framework for bank supervision.

2006 AFRITAC East advisors on finalization of OBL and financial regulations

2007 AFRITAC East advisors on workshops for implementation of OBL and financial regulations

2007 MCM expert on bank restructuring.

2007 MCM expert on foreign exchange operations.

\section{Resident Representative:}

Mr. Lars Holger Engström assumed his duties as Resident Representative in February 2005.

\section{Management Visit:}

The Deputy Managing Director, Mr. Portugal, visited Rwanda during May 3-5, 2007. 


\section{Appendix II. Rwanda: Relations with the World Bank Group}

(As of May 2007)

\section{Partnership for Rwanda's development strategy}

Donor agencies have been key players in Rwanda since the genocide. With the support from the international community, Rwanda has made notable progress along an ambitious path of reconstruction, national reconciliation, and economic reform. In recent years, the Government has made some ambitious efforts, based on its Poverty Reduction Strategy Paper (PRSP), to reduce poverty and improve living conditions of the poor. The PRSP was completed in June 2002. This strategy was supported and discussed by the Boards of the IDA and the IMF on August 12, 2002. The PRSP targeted the halving of poverty by 2015 through a private sector and rural sector strategy. The strategy mainly focuses on six priority areas: (1) rural development and agricultural transformation; (2) human development; (3) economic infrastructure; (4) good governance; (5) private sector development; and (6) institutional capacity building - as the focus for public actions on poverty reduction. Civil society, government agencies and ministries, and donors have all been actively involved in the PRSP process and monitoring. The first PRSP progress report was issued in July 2003 and a BankFund JSA produced in May 2004. The second PRSP progress report was issued in December 2004 and a Bank-Fund JSA was produced in March 2005. The third progress report of the PRSP was issued in July 2005, and the corresponding Bank-Fund JSAN was produced in March 2006.

\section{World Bank Group Program and Portfolio}

The last Country Assistance Strategy for Rwanda was discussed by the World Bank Board in December 2002. The current CAS, in support of the first PRSP, will soon come to an end, and has been extended through the preparation of an Interim Strategy Note (ISN) which was approved by the Board in September 2006. The ISN ensures that the next CAS, to be prepared jointly with DFID, is aligned with the second PRSP. Both the CAS and ISN set out an assistance program consistent with the country's PRSP and emphasize the need to move progressively from project-based approaches to budget support. In line with this approach, a Poverty Reduction Strategy Credit (PRSC) went to the Board in October 2004. This credit would help strengthen GoR capacity to (i) plan and budget results-oriented public sector actions supporting the implementation of the Poverty Reduction Strategy; (ii) develop incentive frameworks through performance-based payments and contracting; (iii) establish strong accountability mechanisms enhancing the capacity of Rwandan citizens to monitor and provide feedback to service providers - both public and private; and (iv) implement a sound fiduciary framework, as well as a monitoring and evaluation system to facilitate transparency and accountability in service delivery for the sectors of focus (i.e., health, education, water, energy). A second programmatic operation, Poverty Reduction Support 
Grant (PRSG), went to the Board in October 2005 and focused on (i) creating a favorable private sector investment climate that would promote sustained economic growth; (ii) improving quality, coverage, and equity of basic service delivery; and (iii) improving public expenditure management and governance. A third PRSG continued with the same areas of focus and went to the Board in December 2006. The Bank has initiated the preparation of a fourth PRSG which could be presented to the Board by the middle of the next fiscal year.

International Development Agency (IDA) Program: Rwanda joined the World Bank in 1963. Since then, the country has been approved for 69 IDA credits and grants totaling approximately US\$1.4 billion. As of March 2007, there were twelve ongoing operations with a commitment value of about US\$347 million and an undisbursed balance of roughly US\$162 million.

Overall, IDA has financed projects in (i) infrastructure, particularly road construction and maintenance, electricity and water supply, and sanitation infrastructure; (ii) agriculture, rural development, and forestry; (iii) social infrastructure, including health and population, and education and training; (iv) private sector development, public enterprise reform, financial development, and technical assistance; and (v) two policy-based quick-disbursing operations (IRC and PRSC1). During the immediate post-genocide period, IDA financed two emergency budget support operations and a social fund-type project, and restructured its prewar portfolio of investment projects to meet the high-priority needs associated with the emergency and the transition from conflict to development.

International Finance Corporation (IFC) Program: The following outlines the IFC program in Rwanda:

- Infrastructure: IFC is working with the World Bank and GOR to explore financing opportunities for the Lake Kivu Methane Gas Project (KPI). IFC has committed US\$7.5 million to M/s Intraspeed SA Rwanda Ltd (ISARL), a major freight and forwarding company in the Great Lakes Region, to help upgrade and expand the company's freight hauling capabilities.

- Financial sector: IFC is considering providing long term credit lines and trade finance products to privatized banks-- Banque Commerciale du Rwanda (BCR) and Bancor.

- Tourism: IFC is considering providing financing (up to US\$7.0 million) for the expansion and modernization of the Serena Hotel in Kigali, and the Kivu Sun in Kibuye, recently acquired by Tourism Promotion Services of the Aga Khan Fund for Economic development (AKFED). IFC is also considering financing the rehabilitation and refurbishment of Hotel Milles de Collines, recently sold to Milkor hotels. 
- Privatization: IFC is advising the Government of Rwanda on the privatization of Rwanda Air.

- Small Investment Prospects in Selected African Countries: Rwanda is one of 6 African countries selected by IFC to participate in a special study to identify prospects for smaller investments. A few leads have been identified.

- PEP-Africa: Technical assistance programs in Financial and SME sectors. Rwanda Leasing Program and Rwanda Entrepreneurship Program were launched on March 15, 2007. IFC is exploring the possibility of launching Gender Entrepreneurship Markets (GEM) in Rwanda.

Multilateral Investment Guarantee Agency (MIGA) Program: Rwanda signed and ratified the MIGA Convention on October 27, 1989. On September 27, 2002, it became a full member of MIGA with the completion of its membership requirements, including payment of the usable currency and the local currency portions of its initial subscription, and deposit of the promissory note. The membership was followed by Rwanda's election to MIGA's Board of Directors during the World Bank/IMF annual meetings held in Washington.

Rwanda is one of the 16 post-conflict countries in the region on which MIGA is focusing as part of its post-conflict strategy. To date, there have been no requests for guarantees coverage of projects in the country, and thus the Agency's efforts have focused on complementing the Bank Group's strategy of accelerating private sector-led growth in the country. In consultation with the Bank, MIGA's technical assistance team undertook an assessment to assist Rwanda in creating a national investment and trade promotion capability and made recommendations on the proposed Rwanda Investment Authority, as well as on the medium to long-term prospects for the country to attract foreign investment. Previously, MIGA collaborated with the World Bank to advise on the establishment of the Rwanda Investment and Export Promotion Agency (RIEPA) and provided considerable information on investment prospects in the country.

In addition, MIGA's on-line investment promotion services (www.fdixchange.com and www.ipanet.net) feature approximately 90 documents on investment opportunities and the related business, legal and regulatory environment in Rwanda.

\section{World Bank staff}

Questions may be referred to Pedro Alba (Tel. 202-458-2246) and Kene Ezemenari (Tel.202-458-5559). 


\section{Table 1: Summary of Bank-Fund Collaboration}

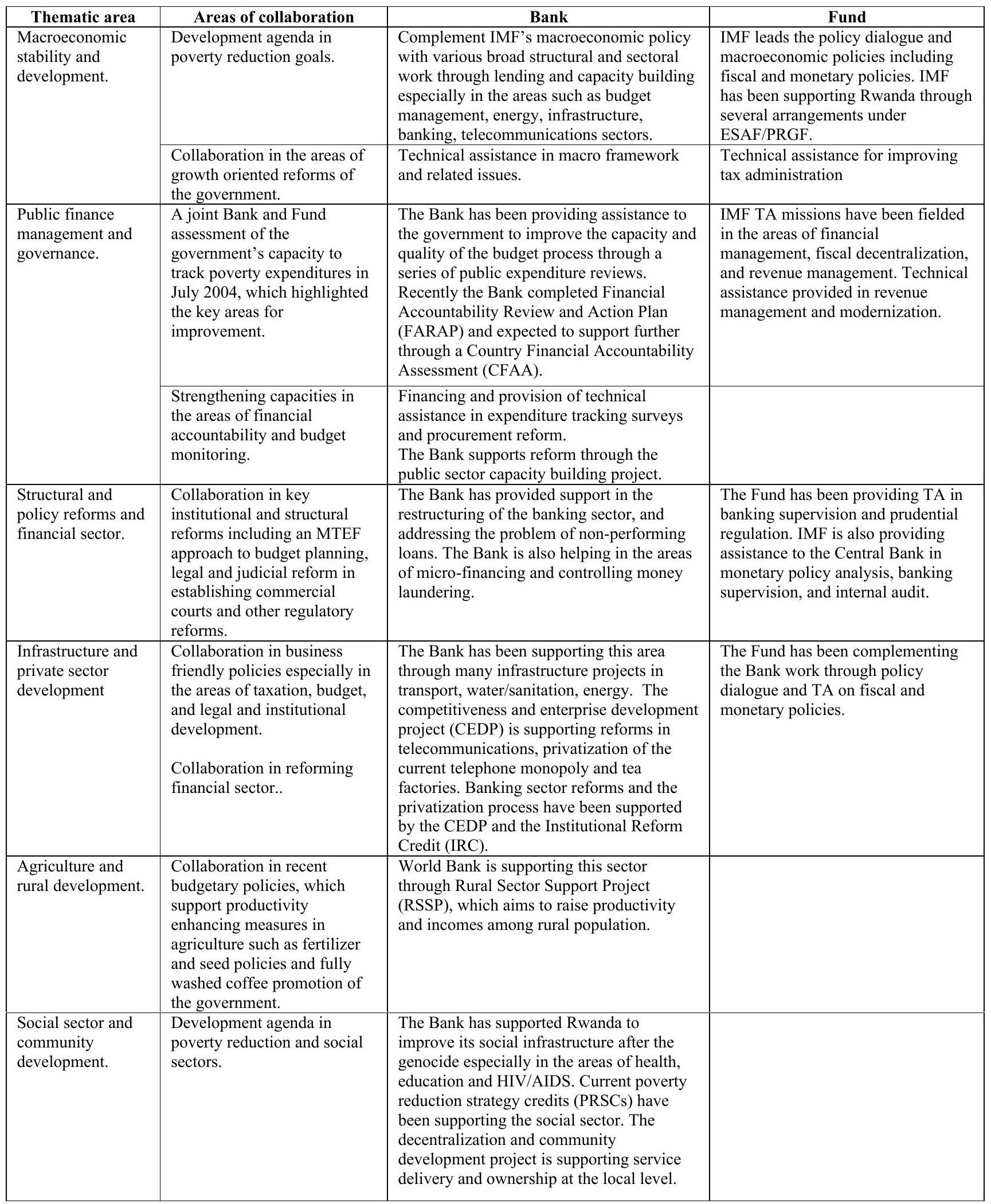


Table 2: Status of Active Operations

\begin{tabular}{|c|c|c|c|c|c|c|}
\hline \multirow[t]{2}{*}{ Project } & \multirow[t]{2}{*}{ US\$ mil. } & \multirow[t]{2}{*}{ Objective } & \multirow{2}{*}{$\begin{array}{c}\text { Approval/ } \\
\text { Effectiveness }\end{array}$} & \multirow{2}{*}{$\begin{array}{l}\text { Disbursed } \\
\text { as of } \\
\text { Mar ' } 07 \\
\end{array}$} & \multicolumn{2}{|c|}{ Ratings } \\
\hline & & & & & DO & IP \\
\hline $\begin{array}{l}\text { Rural Water Supply and } \\
\text { Sanitation }\end{array}$ & 21.67 & $\begin{array}{l}\text { To increasing the availability and } \\
\text { sustainability of water supply and } \\
\text { sanitation (WSS) services in rural areas; } \\
\text { strengthening the capacity of } \\
\text { communities and agencies, and mobilize } \\
\text { community support. }\end{array}$ & $\begin{array}{c}\text { June } 2000 / \\
\text { January } 2001\end{array}$ & 16.63 & $\mathrm{~S}$ & $\mathrm{~S}$ \\
\hline $\begin{array}{l}\text { Human Resources } \\
\text { Development }\end{array}$ & 37.14 & $\begin{array}{l}\text { To build up Rwanda's human resources } \\
\text { and institutional capacity to deliver } \\
\text { education services. }\end{array}$ & $\begin{array}{c}\text { June } 2000 / \\
\text { January } 2001\end{array}$ & 28.29 & $\mathrm{~S}$ & $\mathrm{~S}$ \\
\hline Rural Sector Support & 55.87 & $\begin{array}{l}\text { To build capacity in the management of } \\
\text { farmed marshland and hillside areas; } \\
\text { access to credit and competitiveness in } \\
\text { agricultural export; and small-scale rural } \\
\text { infrastructure. }\end{array}$ & $\begin{array}{l}\text { March 2001/ } \\
\text { October 2001 }\end{array}$ & 37.57 & $\mathrm{~S}$ & $\mathrm{~S}$ \\
\hline $\begin{array}{l}\text { Regional Trade } \\
\text { Facilitation }\end{array}$ & 7.93 & $\begin{array}{l}\text { To improve access to financing for } \\
\text { productive transactions and cross-border } \\
\text { trade. }\end{array}$ & $\begin{array}{l}\text { April 2001/ } \\
\text { August 2001 }\end{array}$ & 5.31 & $\mathrm{~S}$ & $\mathrm{~S}$ \\
\hline $\begin{array}{l}\text { Competitiveness and } \\
\text { Enterprise Development } \\
\text { Project }\end{array}$ & 46.15 & $\begin{array}{l}\text { To establish an enabling environment for } \\
\text { private sector-led economic growth and } \\
\text { poverty reduction in Rwanda. }\end{array}$ & $\begin{array}{c}\text { April 2001/ } \\
\text { December } 2001\end{array}$ & 36.38 & $\mathrm{~S}$ & MS \\
\hline $\begin{array}{l}\text { Rwanda Demobilization } \\
\text { and Reintegration } \\
\text { Project }\end{array}$ & 43.13 & $\begin{array}{l}\text { To help consolidate peace in the Great } \\
\text { Lakes region and foster reconciliation } \\
\text { within Rwanda. }\end{array}$ & $\begin{array}{c}\text { April 2002/ } \\
\text { September 2002 }\end{array}$ & 25.70 & $\mathrm{~S}$ & $\mathrm{~S}$ \\
\hline $\begin{array}{l}\text { Multi-Sectoral } \\
\text { HIV/AIDS }\end{array}$ & 32.46 & $\begin{array}{l}\text { To strengthen prevention measures in } \\
\text { order to slow down the spread of } \\
\text { HIV/AIDS; and expand support and care } \\
\text { for those infected or affected by } \\
\text { HIV/AIDS. }\end{array}$ & $\begin{array}{l}\text { March 2003/ } \\
\text { August 2003 }\end{array}$ & 30.67 & $\mathrm{~S}$ & $\mathrm{~S}$ \\
\hline $\begin{array}{l}\text { Decentralization and } \\
\text { Community } \\
\text { Development }\end{array}$ & 20.53 & $\begin{array}{l}\text { To boost the emergence of a dynamic } \\
\text { local economy, through communities } \\
\text { who are empowered to lead their own } \\
\text { development process under an effective } \\
\text { local government. }\end{array}$ & $\begin{array}{l}\text { June 2004/ December } \\
2004\end{array}$ & 5.70 & $\mathrm{~S}$ & $\mathrm{~S}$ \\
\hline $\begin{array}{l}\text { Public Sector Capacity } \\
\text { Building }\end{array}$ & 19.67 & $\begin{array}{l}\text { To ensure that public sector entities have } \\
\text { the capacity for more efficient, effective, } \\
\text { transparent and accountable performance } \\
\text { in their redefined roles and functions and } \\
\text { for achievement of their strategic } \\
\text { objectives contributing to the } \\
\text { implementation of the PRSP }\end{array}$ & July 2004/ & 3.35 & $\mathrm{~S}$ & MS \\
\hline $\begin{array}{l}\text { Urgent Electricity } \\
\text { Rehabilitation }\end{array}$ & 24.13 & $\begin{array}{l}\text { To alleviate power shortages, and } \\
\text { enhance the capabilities of energy sector } \\
\text { institutions. }\end{array}$ & January 2005/ & 2.0 & MS & MS \\
\hline $\begin{array}{l}\text { Urban Infrastructure and } \\
\text { City Management }\end{array}$ & 21.61 & $\begin{array}{l}\text { To increase access to priority urban } \\
\text { infrastructure in Kigali and two } \\
\text { secondary cities (Ruhengeri and Butare) }\end{array}$ & $\begin{array}{l}\text { November 2005/ } \\
\text { March } 2006\end{array}$ & $3 . .48$ & $\mathrm{~S}$ & $\mathrm{~S}$ \\
\hline $\begin{array}{l}\text { East Africa Trade and } \\
\text { Transport Facilitation }\end{array}$ & 199.53 & $\begin{array}{l}\text { To improve trade environment through } \\
\text { the effective elimination of tariff barriers } \\
\text { in the EAC Customs Union area; } \\
\text { enhance logistics services efficiency } \\
\text { along key corridors by reducing non } \\
\text { tariff barriers and uncertainty of transit } \\
\text { time; and improve railway services in } \\
\text { Kenya and Uganda. }\end{array}$ & $\begin{array}{l}\text { January 2006/ } \\
\text { June } 2006\end{array}$ & 29.58 & $\mathrm{~S}$ & $\mathrm{~S}$ \\
\hline E-Rwanda & 10.00 & $\begin{array}{l}\text { To improve (i) efficiency and } \\
\text { effectiveness of some internal processes } \\
\text { of the Government of Rwanda, and (ii) } \\
\text { the delivery of services in selected key } \\
\text { sectors, including better access to } \\
\text { information through the use of } \\
\text { technology. }\end{array}$ & September 2006/ & 0 & $\mathrm{~S}$ & $\mathrm{~S}$ \\
\hline
\end{tabular}




\section{Appendix III. RWANDa: STATISTICAL ISSUES}

Although economic data are generally adequate for surveillance, weaknesses hamper economic analysis. National accounts, prices, government finance, and balance of payments statistics continue to suffer from significant weaknesses. Monetary statistics and data relevant for banking supervision are adequate for program monitoring, but there is some scope for improvements in quality and timeliness. Rwanda has participated in the General Data Dissemination System since October 2003.

In August 2005, the National Institute of Statistics (NIS) was established following the passage of the new Statistics Law.

\section{National Accounts and Price Statistics}

The national accounts are compiled and disseminated by the NIS, based on the 1968 System of National Accounts methodology. Quality is weak, reflecting inadequate human and material resources. While considerable effort was made to improve the reliability of GDP estimates using the production approach, significant weaknesses in data collection on expenditures and income hinders assessment of savings and investment. The reliability of national accounts estimates is further hampered by weak external sector statistics.

Since 2003, the East AFRITAC has advised the authorities on real sector statistics issues. This assistance is focused on capacity building to enable the construction of short-term indicators such as a monthly PPI for the manufacturing sector, which is a joint project with the central bank (NBR). The results have not yet been integrated in the national accounts. A DFID project is also supporting the NIS with a component on national accounts, aiming to establish a program of economic surveys and the development of leading indicators that can serve as source data for national accounts. The NIS is working on a new benchmarking of GDP estimates (2001). Work has also advanced in the implementation of the 1993 SNA.

The consumer price index (CPI, 2003=100) utilizes expenditure weights derived from a 200001 survey of 6,450 households (local goods account for about 70 percent of expenditure and imported goods about 30 percent; food and drink account for 37 percent of expenditures and housing and energy amount to 16 percent). Certain shortcomings remain, as the CPI aggregates infrequently purchased products in groups with equal weight.

Real sector data are reported regularly for publication in International Finance Statistics (IFS), although with some lag, particularly for GDP estimates. Data on employment and wages are not collected, except for the central government and for daily informal work. 


\section{Government Finance Statistics}

Detailed monthly revenues and expenditures are reported to AFR with a lag of three to four weeks. These data are compiled by the flash-reporting unit of MINECOFIN. A functional classification of government expenditure has been available since the 2003 budget. Within the economic classification, expenditures on PRSP-designated "priority areas" are clearly identifiable. The fiscal data do not capture capital expenditure consistently because capital projects (almost entirely externally financed) are mainly carried out by line ministries outside the regular budget process. Compilation of data on external budgetary assistance as well as on external debt would benefit from strengthened coordination between the finance ministry and the central bank. Efforts are underway to integrate the development budget into the normal budgetary procedures. Fiscal data often showed a discrepancy between deficits and financing estimates. To address these issues, the authorities have made adjustments for changes in the balance of non-core government accounts, changes in cash in vault at the revenue authority, accounting errors, and other factors.

Selected aggregates on annual central government operations through 2004 have been reported to STA for publication in the IFS, but these are subject to large discrepancies, largely due to the timing of recording of expenditures. No sub-annual data are reported to STA, and government finance statistics (GFS) have not been reported for publication in the GFS Yearbook since 1993.

\section{Monetary Statistics}

The balance sheet of the NBR and detailed data on money market transactions are transmitted to AFR on a weekly basis with a lag of one week, while the monetary survey and the consolidated balance sheet of commercial banks are transmitted on a monthly basis with a lag of about five weeks. Detailed data on interbank money market transactions are also provided upon request. Monetary data are reported separately to STA and published in IFS. The data relating to the central bank are provided with a five-week lag, while data on commercial banks are currently reported with a delay of about six months.

The NBR has made progress in the implementation of past TA recommendations. In particular, the NBR adapted in 2004 report forms used by the banking sector so that banks could report data that better reflect the methodology proposed in the Monetary and Financial Statistics Manual. In addition, the NBR is committed to expanding the institutional coverage of the monetary survey to include credit and savings unions and microfinance institutions. The NBR is also pursuing the migration to the Standardized Report Forms (SRFs) for the reporting of its data for dissemination in IFS. Despite these efforts, inconsistencies and problems of timeliness remain in the banking sector data. An STA monetary and financial statistics mission is planned for the second half of 2007 to address outstanding issues and to assist the authorities in completing the migration to standardized reporting. 


\section{External Sector Statistics}

The balance of payments is affected by weaknesses in the collection of source data (treatment of customs data and bank settlement reports, questionnaires) and insufficient staffing. The June 2003 multisector statistics mission recommended: (1) reorganizing data entry and production of external trade statistics, using ASYCUDA and Eurotrace software; (2) adapting survey forms sent to companies to the BPM5 methodology; and (3) collaborating with Central Public Investments and External Finance Bureau (CEPEX) to obtain data on international and bilateral aid. Subsequently, STA balance of payments statistics missions followed up in January 2004 and June 2005, and an AFRITAC mission in October 2006.

Such technical assistance facilitated significant improvements. In particular, the collection of data through direct surveys seems to be well in place, with a satisfactory rate of response, except for embassies. The issue of coverage and availability of the data on external aid to the government and private sector has been solved, thanks to the restructuring of CEPEX (Central Public Investments and External Finance Bureau) and the development of a new database on external financing of the government, which is kept updated by the donors/lenders. Annual balance of payments and IIP data through 2005 have been reported to STA for publication in the IFS. Also, with the assistance of these missions, the NBR has started compiling BOP/IIP statistics in conformity with international standards.

Nevertheless some weaknesses remain, particularly in the compilation of trade data. There are discrepancies between the data on imports and exports published by the NIS and the NBR, which are essentially due to valuation problems. Also, the treatment of bank settlement reports is not effective, because of incomplete automation of the collection of declarations.

Databases on external public debt are maintained by both MINECOFIN and the NBR. A committee, composed of staffs from the ministries of finance and economic planning, foreign affairs, and the NBR, is responsible for collecting, harmonizing, and monitoring information on external public debt. 
Table 3. Rwanda: Table of Common Indicators Required for Surveillance

(As of May 23, 2007)

\begin{tabular}{|c|c|c|c|c|c|}
\hline & 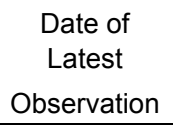 & $\begin{array}{c}\text { Date } \\
\text { Received }\end{array}$ & $\begin{array}{c}\text { Frequency } \\
\text { of } \\
\text { Data }^{6}\end{array}$ & $\begin{array}{l}\text { Frequency } \\
\quad \text { of } \\
\text { Reporting }^{6}\end{array}$ & $\begin{array}{c}\text { Frequency } \\
\text { of } \\
\text { Publication }^{6}\end{array}$ \\
\hline Exchange Rates & Current & Current & D & w & M \\
\hline $\begin{array}{l}\text { International Reserve Assets and Reserve } \\
\text { Liabilities of the Monetary Authorities }{ }^{1}\end{array}$ & Current & Current & W & W & M \\
\hline Reserve/Base Money & 05/11/07 & 05/18/07 & W & W & M \\
\hline Broad Money & 03/31/07 & 05/08/07 & M & M & M \\
\hline Central Bank Balance Sheet & $05 / 11 / 07$ & 05/18/07 & W & W & M \\
\hline $\begin{array}{l}\text { Consolidated Balance Sheet of the Banking } \\
\text { System }\end{array}$ & $03 / 31 / 07$ & 04/08/07 & M & M & M \\
\hline Interest Rates ${ }^{2}$ & 03/31/07 & 05/08/07 & M & M & M \\
\hline Consumer Price Index & $04 / 30 / 07$ & 05/15/07 & M & M & M \\
\hline $\begin{array}{l}\text { Revenue, Expenditure, Balance and Composition } \\
\text { of Financing }^{3}-\text { General Government }\end{array}$ & NA & NA & NA & NA & NA \\
\hline $\begin{array}{l}\text { Revenue, Expenditure, Balance and Composition } \\
\text { of Financing }{ }^{3}-\text { Central Government }\end{array}$ & $12 / 31 / 06$ & 02/14/07 & M & M & M \\
\hline $\begin{array}{l}\text { Stocks of Central Government and Central } \\
\text { Government-Guaranteed Debt }{ }^{5}\end{array}$ & 12/31/06 & 3/31/07 & A & A & A \\
\hline External Current Account Balance & 2006 & $02 / 14 / 07$ & A & SA & A \\
\hline Exports and Imports of Goods and Services & 2006 & $02 / 14 / 07$ & A & A & A \\
\hline GDP/GNP & 2006 & 2/22/07 & A & SA & A \\
\hline Gross External Debt & & & & & \\
\hline
\end{tabular}

${ }^{1}$ Includes reserve assets pledged or otherwise encumbered as well as net derivative positions.

${ }^{2}$ Both market-based and officially-determined, including discount rates, money market rates, rates on treasury bills, notes and bonds.

${ }^{3}$ Foreign, domestic bank, and domestic nonbank financing.

${ }^{4}$ The general government consists of the central government (budgetary funds, extra budgetary funds, and social security funds) and state and local governments.

${ }^{5}$ Including currency and maturity composition.

${ }^{6}$ Daily (D); Weekly (W); Monthly (M); Quarterly (Q); Annually (A); Semi-annually (SA); Irregular (I); Not Available (NA). 
Press Release No. 07/143

FOR IMMEDIATE RELEASE

June 25, 2007
International Monetary Fund

Washington, D.C. 20431 USA

\section{IMF Executive Board Completes Second Review Under the PRGF Arrangement with Rwanda and Approves US\$1.7 Million Disbursement}

The Executive Board of the International Monetary Fund (IMF) today completed the second review of Rwanda's economic performance under a three-year Poverty Reduction and Growth Facility (PRGF) ${ }^{1}$ arrangement. In completing the review, the Board also approved Rwanda's request for a waiver of the non-observance of a performance criterion pertaining to the net credit to the government at end-2006 and a modification of performance criteria for 2007. The completion of the review enables the release of an amount equivalent to SDR 1.14 million (about US $\$ 1.7$ million), bringing total disbursement under the arrangement to SDR 3.42 million (about US\$5.1 million).

The three-year PRGF arrangement for Rwanda was approved by the Executive Board in June 2006 (see Press Release No 06/121) in an amount equivalent to SDR 8.01 million (about US\$12 million).

At the conclusion of the Executive Board's discussion on Rwanda's economic performance, Mr. Murilo Portugal, Deputy Managing Director and Acting Chair, stated:

"The Rwandese Authorities are to be commended for the satisfactory implementation of macroeconomic policies, which are supported under the Poverty Reduction and Growth Facility (PRGF).

"For 2007, the main challenge lies in managing a grant-financed fiscal expansion without jeopardizing macroeconomic stability, avoiding an acceleration in inflation or crowding out of the private sector.

\footnotetext{
${ }^{1}$ The PRGF is the If's confessional facility for low-income countries. PRGF loans carry an annual interest rate of 0.5 percent and are repayable over 10 years with a $5 \frac{1}{2} 2$-year grace period on principal payments.
} 
"The monetary program has been rebased to better reflect developments in money demand. The main task for the Central Bank now is to closely monitor incipient inflationary pressures. "To allow for greater exchange rate flexibility and, in the absence of hedging instruments, the authorities are taking appropriate steps by moving toward an interbank foreign exchange market in coordination with banks and by opening the market to international banks.

“To preserve external debt sustainability and, given Rwanda's small export base and vulnerability to shocks, new sources of grants should be sought in preference to shifting external financing toward loans.

"Looking forward, public financial management reforms must be stepped up to ensure successful decentralization and productive use of funds. Progress in financial sector reform is encouraging and is expected to accelerate with the Financial Sector Development Plan, which is a sound blueprint for building long-term financial markets.

"Given the limited success in reducing poverty in the past few years, the new Poverty Reduction Strategy Papers (PRSP) needs to revamp Rwanda's poverty strategy. It will be essential to improve monitoring of outcome indicators and raise agricultural yields to address rural poverty," Mr. Portugal said. 


\section{Statement by Laurean W. Rutayisire \\ Executive Director for Rwanda \\ June 25, 2007}

\section{I - Introduction}

My Rwandese authorities would like to express their appreciation to staff for the well written set of papers and for the fruitful dialogue they had during the discussions held in Kigali on the second review under the Poverty Reduction and Growth Facility (PRGF). They are in broad agreement with the thrust of issues identified in the staff's report and have consented to the Fund's publication of the report.

My Rwandese authorities would also like to thank the Executive Board and Management for their continued support and advice. Mr. Portugal's visit to Rwanda early last May gave to my authorities the great opportunity to reaffirm their strong commitment to reforms and adequate Fund-supported programs. This visit has also enabled the Deputy Managing Director to gain first-hand experience, not only the achievements made on economic and political fronts, but also of the daunting challenges facing Rwanda in its efforts to sustain growth and fight poverty. Exchanges with representatives of the private sector, civil society organizations, mass media and donors highlighted progress made by Rwanda since the genocide and underscored the strong support to authorities'economic program and reform agenda.

Since June 2006, Rwanda is successfully implementing a three-year PRGF arrangement, in order to continue addressing challenges in achieving higher economic growth and reducing poverty. In line with program objectives, Rwanda's macroeconomic performance continues to be strong, with growth rate higher than expected, inflation declined through April 2007, international reserves substantially exceeded program target in covering 5.5 months of imports at end-2006 and fiscal realizations in line with the targets. As a result, the program and structural reforms remain broadly on track. Indeed, all quantitative performance criteria set for end-2006 were met, except for the limit on net credit to government, due to delays in reimbursements from the African Union for Rwanda's peacekeeping in Darfur. Two structural benchmarks related to issuing accounting instructions and publication of a fertilizer strategy were implemented with delays, owing to capacity constraints and technical difficulties beyond the authorities' control. The third structural benchmark pertaining to the review of the wage structure has been converted into a new benchmark set for September 2007, as technical assistance is needed in carrying out this exercise.

In view of the overall satisfactory program performance and strong resolve to speed up the implementation of structural reforms, my Rwandese authorities request the completion of the second review under the PRGF arrangement and the waiver for the nonobservance of the quantitative performance criterion on net credit to government which was implemented with 
delay due to capacity constraints and modification of performance criteria due to further TA needs. My authorities are determined to achieve the objectives set out in the program meant to preserve macroeconomic stability, advance in reforms, boost growth and strengthen competitiveness.

\section{II - Performance under the Program}

The continued implementation of reforms has led to positive and significant macroeconomic developments in Rwanda. In 2006, despite adverse weather conditions on agricultural sector, economic growth was higher than estimated, reaching more than 5 percent thanks to buoyant activity in the manufacturing and services sectors.

In the fiscal area, performance was strong, reflecting the authorities' determination to implement the fiscal program. The end-2006 target on the domestic fiscal balance was achieved with revenue mobilization higher than anticipated, due mostly to one-off VAT collections and non tax receipts. On the expenditure side, priority spending substantially exceeded the target with additional outlays for schools, water project and export promotion. In order to enhance public financial management, the authorities organized workshops to facilitate the implementation of the Organic Budget Law. They also decentralized the authority to effect expenditure commitments and transfers to budget agencies. In addition, the public procurement code was passed by the parliament and intergovernmental fiscal relations unit was established, with a view to report on local government finances. The audit of the peace keeping activities was also completed. Determined to avoid any delay in the reimbursements from the AU and comply with the limits on net credit to government, the authorities agreed on an appropriate payments' timetable. They stand ready to cut other current expenditure to meet the target in the case of any delay in the reimbursements.

On the monetary front, inflation declined to 10 percent in April 2007 from 12 at end-2006. The international reserves substantially increased to exceed five months of imports. The real exchange rate appreciated by 4 percent and the authorities are concerned about the impact of a strong appreciation on exports. To prevent further appreciation, the authorities issued domestic papers, which helped to bring reserve money within the target set for end-2006. Regarding the current account position, the deficit narrowed more than expected, following continued good policy performances that encouraged private sector's transfers and increase in export receipts.

On the structural front, the authorities have put emphasis on public finance management, poverty reduction, financial sector reform and private sector development. In this regard, it is worth noting that a national fertilizer strategy was developed and published in February aiming at increasing agricultural productivity to improve food security and reduce poverty in rural areas. A financial sector development plan was prepared and presented to donors for financing. The foreign exchange regulations were revised in addition to relaxing the auctions rules, in order to promote the foreign exchange market. 


\section{III - Outlook and Policies for 2007}

The macroeconomic policy framework for 2007 remains consistent with the authorities' objectives over the medium-term, namely ensuring broad-based economic growth to sustain poverty reduction and enhance Rwanda's external competitiveness. As stated in the staff report, prospects for scaling up of aid are positive. The authorities are committed to the most productive use of resources with a view to preserve macroeconomic stability, accelerate growth and sustain poverty reduction.

In 2007, economic growth is projected to be in the range of 4.5 and 6.5 percent. Inflation is expected to decline to 5 percent, while international reserves would cover about 5 months of imports. The authorities intend to press ahead with their enhancement of public finance management and remove obstacles to private sector.

\section{Fiscal Policy}

The Rwandese authorities are strongly committed to implement prudent fiscal policy, notably in the context of substantial fiscal expansion resulting from a scaling up of aid. They will focus on preventing a crowding out of the private sector as well as building up of onerous domestic debt and rekindling inflation. In this regard, indicators on domestic fiscal balance and net credit to government will be closely monitored. On the revenue side, the revenue-toGDP ratio will be maintained at 14.7 percent. In the case of revenue shortfalls, the authorities stand ready to increase the petroleum excise. As for expenditure, all new spending is for priority sectors, notably education, food security, Lake Kivu methane gas pilot project, infrastructure repairs and emergency assistance to flood victims. The action plan for the medium-term public financial management will be completed by end-2007. This plan designed to ensure full implementation of the organic budget law and financial regulations includes measures to improve on public accounts, budget preparation, budget execution and capacity building in the office of the Auditor General and the Internal Audit Department, through the technical assistance from the World Bank and AFRITAC. The diagnostic review of the Medium-Term Expenditure Framework covers the integration of the development and recurrent budget.

\section{Monetary Policy and Financial Sector Reform}

Monetary policy will continue to aim at achieving the inflation objective set for 2007. To this end, reserve money will remain the operational target and monetary management will be improved. The authorities intend to rebase the reserve money program and monitor its developments on a monthly basis. The growth of money reserve will be limited to 13 percent and further room for an expansion of credit to private sector. The management of excess reserves of banks will be tightened through the sales of foreign exchange to encourage a stronger interbank market. With these measures, inflation is expected to be reduced at 5 percent. In their efforts to enhance the exchange rate's flexibility, the authorities will engage 
with banks in the preparations for an exit from the current auction system toward a fullyfledged interbank market.

The authorities will continue to enhance progress made in the financial sector reform. To improve banks' capital base and tighten the licensing criteria, the minimum capital requirement for banks will be increased as of January 2008. Disinvestment of the Banque of Kigali is underway and during the transition period, an interim management was established. Moreover, the Financial Sector Development Plan (FSDP) includes strengthening the NBR's supervisory functions, expanding access to credit and financial services, notably outside the capital, developing long-term finance and capital markets, consolidating regulation and supervision for nonbank financial institutions namely insurance companies and pension funds. Regarding the modernization of the payments system, the authorities aim at developing a national strategy on payments and establishing an automated clearing house. On the AntiMoney Laundering front, the authorities will establish a Financial Intelligence Unit to enforce the law, following its adoption by the Parliament.

\section{Structural Reforms and Competitiveness}

My Rwandese authorities are aware of the challenges facing the economy and the need to pursue implementation of their reform agenda. Sustaining economic growth and improving the country's competitiveness require in particular boosting the agricultural productivity, finding lasting solution to the energy difficulties and improving the conditions of the private sector development. Remarkable progress has been achieved with regard to the public finance management and financial sector reform. The authorities are exploring various options to increase the electricity supply, including the Lake Kivu methane gas pilot project and a heavy-fuel oil generator. Electrogaz's management was strengthened and its financial situation is expected to improve in the medium-term. A structured tariff has been introduced and further adjustments are scheduled. Reforms in the Justice and land sectors were accelerated, in order to improve the business environment. In this respect, a three-year project in partnership with the private sector was launched last May, with a view to address legal and regulatory constraints facing investors and businesses. In the same vein, the authorities will speed up their efforts to invest in infrastructure and promote new information technologies and communication networks to further attract private investment.

As regards the regional trade and integration, the Rwandese authorities are developing an implementation roadmap for joining by June 2009 the East African Community's Customs Union Protocol.

With regard to poverty reduction, my authorities are reviewing policies and outcome indicators in updating the PRSP. They will intensify their efforts to tackle extreme poverty by improving food security and fostering job creation. The revised strategy will focus on schemes to raise agricultural productivity and address population issues, including growth rate. As poverty remains concentrated in rural areas, increasing agricultural productivity is 
crucial. Accordingly, the agricultural strategy will be finalized in time to enable its implementation with the 2008 budget.

On debt sustainability, the authorities remain committed to pursuing prudent debt management.

\section{IV - Conclusion}

My Rwandese authorities have continued to demonstrate their strong commitment to implementing sound policies and reforms, which have resulted in appreciable economic performances. Based on the satisfactory program implementation, I would appreciate Directors support of my authorities' request for the completion of the second review under the PRGF arrangement and waiver of nonobservance of performance criterion and modification performance criteria. The Rwandese authorities thank the international community and the Fund for their continued assistance. They remain committed to achieve the objectives and implement policies set forth under the PRGF arrangement. 